\title{
GCU
}

Glasgow Caledonian

University

University for the Common Good

\section{Fin control for ship roll motion stabilisation based on observer enhanced MPC with disturbance rate compensation}

Jimoh, Isah Abdulrasheed; Kucukdemiral, Ibrahim Beklan; Bevan, Geraint

Published in:

Ocean Engineering

DOI:

10.1016/j.oceaneng.2021.108706

Publication date:

2021

Document Version

Author accepted manuscript

Link to publication in ResearchOnline

Citation for published version (Harvard):

Jimoh, IA, Kucukdemiral, IB \& Bevan, G 2021, 'Fin control for ship roll motion stabilisation based on observer enhanced MPC with disturbance rate compensation', Ocean Engineering, vol. 224, 108706.

https://doi.org/10.1016/j.oceaneng.2021.108706

\section{General rights}

Copyright and moral rights for the publications made accessible in the public portal are retained by the authors and/or other copyright owners

and it is a condition of accessing publications that users recognise and abide by the legal requirements associated with these rights.

Take down policy

If you believe that this document breaches copyright please view our takedown policy at https://edshare.gcu.ac.uk/id/eprint/5179 for details

of how to contact us. 


\section{Ocean Engineering \\ Fin control for ship roll motion stabilisation based on observer enhanced MPC with disturbance rate compensation \\ --Manuscript Draft--}

\begin{tabular}{|c|c|}
\hline Manuscript Number: & OE-D-20-02467R2 \\
\hline Article Type: & Full length article \\
\hline Keywords: & $\begin{array}{l}\text { Ship Roll Stabilisation; Fin Stabiliser; Model Predictive Control; Disturbance } \\
\text { Rejection; Disturbance Rate Compensation }\end{array}$ \\
\hline Corresponding Author: & $\begin{array}{l}\text { Isah Abdulrasheed Jimoh } \\
\text { Glasgow Caledonian University } \\
\text { Glasgow, Scotland UNITED KINGDOM }\end{array}$ \\
\hline First Author: & Isah Abdulrasheed Jimoh, BEng, MSc \\
\hline \multirow[t]{3}{*}{ Order of Authors: } & Isah Abdulrasheed Jimoh, BEng, MSc \\
\hline & Ibrahim B Küçükdemiral, BSc, MSc and PhD \\
\hline & Geraint Bevan, PhD \\
\hline Abstract: & $\begin{array}{l}\text { In this paper, a disturbance observer enhanced model predictive controller (MPC) } \\
\text { which compensates for the wave-induced disturbance magnitude and rate is proposed } \\
\text { for the ship roll motion stabilisation problem. The velocity model of the ship roll motion } \\
\text { is used in the controller design to handle slowly varying modelling uncertainties and } \\
\text { external disturbances. The rate of change of the disturbances induced by waves is } \\
\text { then attenuated by formulating a control input that incorporates the estimated } \\
\text { disturbance rate such that it is always in opposition to the rate of the environmental } \\
\text { disturbances. The disturbance estimation was achieved by designing an observer } \\
\text { based on convex optimisation formulated as an } \mathrm{H} 2 \text { minimisation problem. } \\
\text { Numerical simulation studies, under various conditions of the sea, showed the } \\
\text { effectiveness of the proposed MPC scheme in reducing the undesired ship roll motion } \\
\text { induced by sea waves. }\end{array}$ \\
\hline
\end{tabular}




\title{
Fin control for ship roll motion stabilisation based on observer enhanced MPC with disturbance rate compensation
}

\author{
Isah Abdulrasheed Jimoh*, Ibrahim Beklan Küçükdemiral, Geraint Bevan \\ School of Computing, Engineering and Built Environment, Glasgow Caledonian University, 70 Cowcaddens \\ Rd, G4 OBA, Glasgow, UK.
}

\begin{abstract}
In this paper, a disturbance observer enhanced model predictive controller (MPC) which compensates for the wave-induced disturbance magnitude and rate is proposed for the ship roll motion stabilisation problem. The velocity model of the ship roll motion is used in the controller design to handle slowly varying modelling uncertainties and external disturbances. The rate of change of the disturbances induced by waves is then attenuated by formulating a control input that incorporates the estimated disturbance rate such that it is always in opposition to the rate of the environmental disturbances. The disturbance estimation was achieved by designing an observer based on convex optimisation formulated as an $\mathcal{H}_{2}$ minimisation problem. Numerical simulation studies, under various conditions of the sea, showed the effectiveness of the proposed MPC scheme in reducing the undesired ship roll motion induced by sea waves.
\end{abstract}

Keywords: Ship Roll Stabilisation, Fin Stabiliser, Model Predictive Control, Disturbance Rejection, Disturbance Rate Compensation

\section{Introduction}

Roll stabilisation is one of the most studied problems in ship control because of the danger posed by rolling motions that are induced by ocean waves in high seas. The danger posed by the wave-induced motions threatens the comfort of crew members and overall cargo safety. Hence, measures are usually taken from the design stage to achieve ship roll stabilisation and

${ }^{\star}$ Corresponding author.

Email address: ijimoh200@caledonian.ac.uk (Isah Abdulrasheed Jimoh). 
this generally involves increasing the natural roll period of ships. Perez (2006) pointed out that despite efforts geared towards extending the natural rolling period of the vessel, waveinduced motion is always present under certain sailing conditions. To ensure safe operation of vessels in these conditions, several roll stabilisation devices including antiroll tanks, rudder, fins, bilge keels, etc. and their associated control systems have been developed (Bassler and Reed, 2009; Irkal et al., 2019; Li et al., 2016; Liu et al., 2020). Comprehensive reviews of the development of stabilising control design for ship roll motion were done by Perez (2006) and Kula (2015).

Active fin stabilisers are widely used in practical applications of roll motion stabilisation especially in high-speed vessels and are very attractive because of their good performance in roll motion reduction and relatively easy control design (Perez, 2006). Simple PID controllers developed using classical control theory can be used to stabilise roll using active fins (Surendran et al., 2007). Although these controllers are easy to design and implement, they can only be used under restricted environmental conditions and for a restricted class of ships (Crossland, 2003). This is because a controller that cannot adapt to changes in environmental conditions resulting in nonlinear dynamics may result in roll motion amplification instead of reduction (Perez and Blanke, 2012). Moreover, there is a need to consider system constraints during design since it is usually desired to ensure that the amplitude and rate of the fin actuator and even that of the effective angle of attack of the fins do not exceed a certain threshold angle and angular velocity (Liu et al., 2011; Perez and Goodwin, 2008).

In handling the challenges posed by the wave-induced nonlinear dynamics, model uncertainties, and external disturbances in roll dynamics, different advanced control schemes have been proposed. Sharif et al. (1995) proposed a multivariable approach where fin and rudder were simultaneously considered for the reduction of wave-induced roll motions. Although the approach results in good anti-rolling fin performance, it imposes strict requirement on the rudder which induces significant wearing of the device. In Hickey et al. (1997, 1995), $H_{\infty}$ design approach was proposed for roll stabilisation. Hinostroza et al. (2015) employed $H_{\infty}$ control to stabilise roll-fin dynamics where $L_{2}$ gain was used to guarantee the robustness of the controller. The challenge with the methods is that the fin actuator constraints were not considered. More recently, Kuseyri (2020) used $H_{\infty}$ control in ship roll stabilisation, 
however, the method was based on a gyroscopic device. Fuzzy logic based controllers have also been proposed for fin stabilisers (Surendran and Kiran, 2007; Sutton et al., 1990). The control method was used for time-delay ship roll stabilisation in (Bai, 2014). Other intelligent controllers based on neural networks (NNs) have also been developed for ship roll motion stabilisation using fin stabilisers (Bai et al., 2013; Li et al., 2016; Wang et al., 2012). Although Li et al. (2016) considered output constraint, the controllers generally fail to consider the physical limitation of the fin angle magnitude and rate. Sun et al. (2018) used a Radial Basis Function NN approach to design an adaptive control for lift-feedback system of fin stabilisers in order to prevent anti-rolling. At this point, it is important to note that for a controller to guarantee the safety of a dynamic system, it should be deterministic so that it is possible to validate safe operation under all conditions. Since neural network-based control schemes can be stochastic in nature, it is important to put this into consideration when designing such controllers for roll reduction since sailing conditions vary significantly.

Obtaining optimum performance in the control of dynamic systems is usually desirable. In the stabilisation of ship roll motion, linear quadratic regulator (LQR) and model predictive control (MPC) algorithms are the two main optimal control methods used. Fortuna and Muscato (1996) proposed an adaptive linear quadratic (LQ) compensator that achieves adaptation via a multilayer perceptron neural network. Pascoal et al. (2005) proposed a linear quadratic regulator feedback mechanism that is coupled with disturbance feedforward while Lee et al. (2011) combined an LQR controller with pod propeller. In addition to providing optimal or sub-optimal performance and effective handling of nonlinearities, MPC provides a more natural approach to handle system constraints when compared to other control schemes (including LQR) that have been proposed for ship roll motion stabilisation. This is due to the fact that the control action is computed by considering the constraints conditions. Control schemes such as LQR and PID, where saturation blocks are used to implement the input/rate constraints, would generally result in higher oscillations when input/rate saturation occurs. This is because the planning of the control action by the controllers does not put the constraints into consideration. Malekizade et al. (2016) developed an MPC scheme based on linear matrix inequalities (LMIs) with operational constraints for the reduction of roll angle and roll rate of ships via fin stabilisers. Perez and Goodwin (2008) 
developed a constrained MPC for fin stabiliser to prevent dynamic stall. In the work, MPC was also used to obtain an adaptive strategy to deal with variation in sailing conditions and sea states by developing a wave disturbance model to predict wave-induced motions which were then embedded in the MPC framework. Perez and Goodwin (2008) then updated the disturbance model whenever sailing conditions changes or every 20 minutes if no change was recorded. Liu et al. (2011) noted that the rate of update is not suitable for fast ferries and naval vessels and hence, they proposed a method that uses a wave-induced force model rather than the wave-induced motion model. They then employed an auto-regressive model for adaptive wave disturbance identification to avoid performance degradation resulting from model uncertainties. None the less, the effectiveness of the controllers (Liu et al., 2011; Perez and Goodwin, 2008) rely on the accuracy of the wave disturbance model which is difficult to precisely model in practice. Moreover, the accuracy of the disturbance prediction is depleted by ship modelling uncertainties even though this effect is more pronounced in the superposition approach of Perez and Goodwin (2008).

In this paper, a velocity model based MPC is proposed for roll motion stabilisation. Although Kucukdemiral et al. (2019) provided detailed mathematical modelling of the vertical motion of a ship, they used the traditional velocity form MPC for irregular waves disturbance rejection which appears naive since constant or slowly-varying disturbances are assumed in the controller formulation. The proposed velocity form in this work is enhanced by a disturbance observer for the compensation of environmental disturbances affecting the ship roll motion. For offset-free model-based predictive control in the presence of modelling errors and external disturbances, recent findings (Jimoh et al., 2020; Pannocchia et al., 2015) have established that the velocity model is equivalent to particular choices of the general approach - disturbance model plus observer. To improve the performance of the conventional velocity model, estimated disturbance increment is incorporated into the control law to further reduce the effect of the actual wave disturbance increment. To achieve this, a control signal with two degrees of freedom is employed by modifying MPC cost function to include a term that mimics disturbance velocity. The computed optimal disturbance velocity allows for the adjustment of the control signal to include the estimated wave disturbance increment such that it is always in opposition to the velocity of the actual wave disturbances. To estimate 
the unmeasurable wave input disturbances, a combined state and disturbance observer similar to that proposed in Pannocchia and Rawlings (2003) is designed. The proposed dynamic observer gain is obtained by solving an appropriate discrete-time $\mathcal{H}_{2}$ minimisation problem. Therefore, the main contributions of this paper to the literature are summarised as follows:

- An MPC algorithm that attenuates the rate of change of the wave-induced disturbances is proposed for ship roll motion stabilisation. To ensure that modelling errors and magnitude of the disturbances have minimum impact on the control method, the incremental form of the ship model is used such that constant or slowly varying uncertain parameters are eliminated in each sampling instant.

- The proposed predictive controller relies on a single-step estimation of the waveinduced disturbance and avoids the need for a wave model based disturbance prediction as in the MPC proposed by Perez and Goodwin (2008) and Liu et al. (2011) for roll motion stabilisation.

- The proposed controller for roll stabilisation was shown to be robustly asymptotically stable since the method reduces the uncertain disturbances to a compact and bounded set.

This paper is organised as follows. Section 2 presents the mathematical nonlinear model of the roll dynamics and the velocity form representation. In Section 3, the controller design details including constraints handling are given. In Section 4, observer design is given for disturbance induced by waves estimation. Section 5 gives an analysis of the stability of the proposed controller and numerical simulation is reported in Section 6 to demonstrate the effectiveness of the control scheme. The numerical simulation results are discussed in Section 7 while concluding remarks are given in the last section.

Notations. In describing a symmetric matrix $X, X \prec 0$ and $X \succ 0$ represent negative definite and positive definite, respectively. Similarly, $X \preceq 0$ and $X \succeq 0$ indicates that $X$ is respectively negative semi-definite matrix and positive semi-definite matrix. The notation $(\cdot)^{T}$ is used to denote the transpose of matrix or vector $(\cdot)$, and $\operatorname{tr}(\cdot)$ represents the trace of a square matrix $(\cdot)$. A matrix element denoted $\star$ implies that it is the transpose of the 
corresponding symmetric element. $A \triangleq B$ means the definition of $A$ is $B$. $A$ is directly proportional to $\mathrm{B}$ is written as $A \propto B$ and blkdiag $\left\{x_{1}, \ldots, x_{N}\right\}$ returns a block-diagonal matrix. $I_{n}$ represents an identity matrix of size $n \times n$. $a \leq b$ describes a component-wise inequality between vectors $a$ and $b . \mathbb{N}$ is the set of natural numbers, $\mathbb{N}_{+}$is the set of positive natural numbers and $\mathbb{R}$ denotes the set of real numbers. $\mathbb{R}^{n}$ shows a $n$-dimensional real vector and $\mathbb{R}^{n \times m}$ represents a $n \times m$ real matrix.

\section{Preliminaries and problem formulation}

\subsection{Description of the ship roll dynamics}

The general model describing the motion of a marine vessel takes into consideration surge, sway, heave, roll, pitch and yaw. In practice, the six degree-of-freedom (6DOF) model is usually reduced. In the study of roll motion dynamics, $4 \mathrm{DOF}$ or $1 \mathrm{DOF}$ is often used (Hinostroza et al., 2015). The 1DOF model considers only the roll motion dynamics and this model will be used in this study as in many previous studies (Hinostroza et al., 2015; Liu et al., 2011; Perez and Goodwin, 2008). In this paper, we will consider the nonlinear model of a ship with some simplifications by neglecting the fifth-order term of the roll angle as in Surendran et al. (2007). This form of simplification was also adopted in Li et al. (2016) and by defining $x=[\phi p]^{T}$, the nonlinear model can be described as follows:

$$
\left\{\begin{array}{l}
\dot{\phi}=p \\
\dot{p}=f(\phi, p)+b \alpha+M_{W} \\
y=x
\end{array}\right.
$$

where $\phi[\mathrm{rad}]$ is the roll angle, $p=\dot{\phi}[\mathrm{rad} / \mathrm{s}]$ is the roll rate and $\alpha[\mathrm{rad}]$ is the fin stabiliser steering angle, that is, the control input. The ship roll dynamic is represented by $f(\phi, \dot{\phi})$, $M_{W}$ is the wave-induced moments acting on the ship per unit inertia moments and $b \alpha=M_{C}$ denotes the control moments per unit inertia moments resulting from the fin stabiliser. The fin stabiliser generated control moment per unit inertia moments can be explicitly given as

$$
M_{C}=-\frac{\rho U^{2} A_{f} l_{f} C_{L}^{\alpha}}{I_{X X}+J_{X X}} \alpha,
$$


while the ship roll dynamics are given by

$$
f(\phi, \dot{\phi})=a_{1} \phi+a_{2} \phi^{3}+a_{3} \dot{\phi}+a_{4} \dot{\phi}|\dot{\phi}|,
$$

where

$$
\begin{aligned}
& a_{1}=-\frac{T h}{I_{X X}+J_{X X}}, a_{2}=\frac{T h}{\phi_{v}^{2}\left(I_{X X}+J_{X X}\right)}, \\
& a_{3}=-\frac{D_{N}}{I_{X X}+J_{X X}}-\frac{\rho U^{2} A_{f} l_{f}^{2} C_{L}^{\alpha}}{\left(I_{X X}+J_{X X}\right) U}, a_{4}=-\frac{D_{W}}{I_{X X}+J_{X X}}, \\
& D_{N}=\frac{2 n_{1} \sqrt{T h\left(I_{X X}+J_{X X}\right)}}{\pi}, D_{W}=\frac{3 n_{2}\left(I_{X X}+J_{X X}\right)}{4}, \\
& I_{X X}+J_{X X}=\frac{T B_{s}^{2}}{g}\left(0.3085+\frac{0.0227 B_{s}}{d}-\frac{0.00043 L}{100}\right)^{2},
\end{aligned}
$$

and $I_{X X}$ and $J_{X X}$ are the mass moment of inertia for roll and the added mass moment of inertia, respectively (alternatively referred to as the inertia moments). $D_{N}$ and $D_{W}$ are the linear and nonlinear damping moments of roll motion. $T$ [tonne] is the displacement of the ship; $h[\mathrm{~m}]$ denotes the initial metacentric height; $\phi_{v}[\mathrm{rad}]$ is the ship flooding angle; $L[\mathrm{~m}]$ is the ship's length between its perpendiculars; $B_{s}[\mathrm{~m}]$ is the width of the ship; $d_{s}[\mathrm{~m}]$ is the ship's draught; $n_{1}$ and $n_{2}$ are test coefficients. The acceleration due to gravity is denoted $g\left[\mathrm{~m} / \mathrm{s}^{2}\right] ; \rho\left[\mathrm{kg} / \mathrm{m}^{3}\right]$ is water density; $U[\mathrm{~m} / \mathrm{s}]$ is forward ship speed; $C_{L}^{\alpha}$ is the rate of change of lift coefficient with respect to $\alpha ; A_{f}\left[\mathrm{~m}^{2}\right]$ is the fin area; $l_{f}[\mathrm{~m}]$ represents the moment arm of the fin stabiliser.

Although Li et al. (2016) considered the nonlinear model described by (1), they considered only output constraints; however, it is arguable that the input and rate constraints are more important because the fin angle and angular velocity which determines the effectiveness of the controller is limited in practice.

\subsection{Wave disturbance model}

The standard practice in the modelling of wave-induced disturbances acting on a ship is to model the sea waves as a stochastic process (Fossen, 2011). This method characterises the sea waves frequency by a power spectral density (PSD). In this paper, the wave spectrum formulated by Pierson Jr and Moskowitz (1964) is employed. The wave spectral formulation was conceived for fully developed wind-generated seas in the North Atlantic Ocean as follows: 


$$
\omega_{0}=\left(\frac{4 B_{w v}}{5}\right)^{1 / 4}
$$

Equation (5) shows that different significant wave heights $H_{s}$ corresponds to different modal frequency $\omega_{0}$ and therefore, different peak values of $S(\omega)$. The wave model can be approximated by a second-order system in the state-space form:

$$
\begin{gathered}
{\left[\begin{array}{c}
\dot{d} \\
\dot{d^{w}}
\end{array}\right]=\left[\begin{array}{cc}
0 & 1 \\
-\omega_{0}^{2} & -2 \zeta_{w} \omega_{0}
\end{array}\right]\left[\begin{array}{c}
d \\
d^{w}
\end{array}\right]+\left[\begin{array}{c}
0 \\
k_{w}
\end{array}\right] w_{n}} \\
y_{w}=\left[\begin{array}{ll}
0 & 1
\end{array}\right]\left[\begin{array}{c}
d \\
d^{w}
\end{array}\right]
\end{gathered}
$$

where $w_{n}$ is a zero mean white process noise, $\zeta_{w}$ is a damping coefficient which may be set to a constant value. A typical value for the damping coefficient is 0.1 (Wang et al., 2019). It is necessary to note that the wave-induced disturbances in (1) is given by $M_{W}=d^{w}$. Furthermore, $k_{w}=2 \zeta_{w} \omega_{0} \sigma_{w}$ where $\sigma_{w}$ is a constant describing the intensity of the wave. The parameter $\sigma_{w}$ can be calculated as the square root of $S\left(\omega_{0}\right)$. Furthermore, for a ship moving with forward speed $U$, the peak frequency of the wave spectrum will be modified according to the following:

$$
\omega_{e}=\omega_{0}-\frac{\omega_{0}^{2}}{g} U \cos \beta
$$

where $\omega_{e}[\mathrm{rad} / s]$ is the encounter frequency and $\beta(\mathrm{rad})$ is the (encounter) angle between the heading and the direction of the wave. Equation (8) implies that the peak frequency of a wave spectrum moving at a forward speed $U>0$ should be modified to $\omega_{e}$ - the encounter frequency. 


\subsection{Velocity model formulation}

This paper aims to design a linear discrete-time MPC for the stabilisation of the nonlinear, continuous-time model of ship roll motion in (1). To achieve this, the nonlinear model (1) is linearised around the operating points $\phi(0)=0 \mathrm{deg}$ and $\dot{\phi}(0)=0 \mathrm{deg} / \mathrm{s}$ and the resulting linear model is sampled at a rate denoted $T_{s}$, the sampling period. The appropriate choice of $T_{s}$ will depend on the type of ship, and in particular its forward speed or typical rate of change of direction. Hence, a discrete-time state space representation can be obtained as follows:

$$
\begin{aligned}
x_{k+1} & =A x_{k}+B u_{k}+B_{d} d_{k}^{w}, \\
y_{k} & =C x_{k}+D d_{k}^{w} .
\end{aligned}
$$

For convenience, we define $p=\dot{\phi}$. Thus, $x_{k}=\left[\phi_{k} p_{k}\right]^{T} \in \mathbb{R}^{n_{x}}$ and $y_{k}=\left[\phi_{k} p_{k}\right]^{T} \in \mathbb{R}^{n_{y}}$ is the measured output, $u_{k}=\alpha_{k} \in \mathbb{R}^{n_{u}}, d_{k}^{w} \in \mathbb{R}^{n_{d}}$ is the wave-induced disturbance; $A, B, C$ are system matrices with appropriate sizes and $B_{d}$ is the disturbance input matrix and $D=0$ since there are no measured disturbances. To deal with the model (9) uncertainties, the velocity form (with delayed output) of the state space model is used and it is given as follows (Jimoh et al., 2020):

$$
\begin{gathered}
{\left[\begin{array}{c}
\sigma_{k+1} \\
y_{k}
\end{array}\right]=\overbrace{\left[\begin{array}{ll}
A & 0 \\
C & I
\end{array}\right]}^{\tilde{A}} \overbrace{\left[\begin{array}{c}
\sigma_{k} \\
y_{k-1}
\end{array}\right]}^{\tilde{x}_{k}}+\overbrace{\left[\begin{array}{c}
B \\
0
\end{array}\right]}^{\tilde{B}} \mu_{k}+\overbrace{\left[\begin{array}{c}
B_{d} \\
0
\end{array}\right]}^{\tilde{B}_{d}} \delta_{k},} \\
y_{k}=\overbrace{\left[\begin{array}{ll}
C & I
\end{array}\right]}^{\tilde{C}}\left[\begin{array}{c}
\sigma_{k} \\
y_{k-1}
\end{array}\right]+\overbrace{D}^{\tilde{D}} \delta_{k} .
\end{gathered}
$$

where $\sigma_{k} \triangleq x_{k}-x_{k-1}$ is the state increment, $\mu_{k} \triangleq u_{k}-u_{k-1}$ is the change in control signal and the disturbance increment $\delta_{k} \triangleq d_{k}^{w}-d_{k-1}^{w}$. The augmented model (10) would be able to eliminate steady state offset due to model mismatch provided that the disturbances are constant or slowly-varying. However, the external wave-induced disturbances $d_{k}^{w}$ may vary significantly such that $\delta_{k} \neq 0$. Therefore, the main objective of this paper is to provide robustness in the presence of model uncertainties and to minimise the impacts of the varying wave-induced disturbances while fulfilling the system constraints. 


\section{Controller design}

The model-based predictive control problem considering the objectives stated in the previous section can be posed as a constrained tracking control problem. Although the roll motion stabilisation problem is a regulation problem that can sufficiently be controlled by a simple output feedback problem, the tracking control formulation is presented because it is more general and will make it easy for the proposed control to be applied to other systems.

Definition 3.1. (Tracking constrained control problem) Given the initial condition $\tilde{x}_{0}$, the previous control $u_{k-1}$ and the desired reference $r_{k}$, find the control action $u_{k}=u_{k-1}+\kappa_{N}$, where $\kappa_{N}: \mathbb{R}^{n_{x}} \mapsto \mathbb{R}^{n_{u}}$ is the incremental control obtained by minimising the objective function:

$$
\min _{\mu, \nu} J=\frac{1}{2} e_{t+N}^{T} S e_{t+N}+\frac{1}{2} \sum_{k=0}^{N-1}\left(e_{t+k}^{T} Q e_{t+k}+\mu_{t+k}^{T} R \mu_{t+k}+\nu_{t+k}^{T} P \nu_{t+k}\right),
$$

Subject to:

$$
\begin{aligned}
\tilde{x}_{t} & =\tilde{x}_{0} \\
\tilde{x}_{k+1} & =\tilde{A} \tilde{x}_{k}+\tilde{B} \mu_{k}+\tilde{B}_{d} \nu_{k} \\
y_{k} & =\tilde{C} \tilde{x}_{k}+\tilde{D} \delta_{k} \\
\left|u_{k}\right| & \leq u_{\max } \\
\left|\mu_{k}\right| & \leq \mu_{\max } .
\end{aligned}
$$

where $(\cdot)^{T}$ denotes the transpose $(\cdot) ; e_{k}=r_{k}-y_{k}$ is the output error; $u_{\max }=\alpha_{\max }$ is the largest mechanical angle the fins can turn through; $\mu_{\max }$ is the maximum fin rate the machinery commanding the fins is allowed to apply. The added term, $\nu_{k}$ is included to mimic the external disturbance increment and it is referred to as 'optimal disturbance increment' which is computed from the optimisation problem. The weights $Q$ and $S$ are positive semidefinite matrices while $R$ and $P$ are positive definite.

If the weighting matrices are selected as

$$
Q=\left[\begin{array}{cc}
q_{\phi} & 0 \\
0 & q_{p}
\end{array}\right], S=\left[\begin{array}{cc}
s_{\phi} & 0 \\
0 & s_{p}
\end{array}\right], R=r_{\mu}, P=p_{\nu}
$$


and $\phi_{r}$ and $p_{r}$ are used to represent the reference values of $\phi$ and $p$ respectively. If $h(x)$ represents a quadratic function of $x$, the cost function can be interpreted as follows:

$$
V \propto s_{\phi} h\left(\phi-\phi_{r}\right)+s_{p} h\left(p-p_{r}\right)+q_{\phi} h\left(\phi-\phi_{r}\right)+q_{p} h\left(p-p_{r}\right)+r_{\mu}(\mu)+p_{\nu} h(\nu)
$$

From (14), it can be drawn that whenever $p_{\nu} \gg r_{\mu}$, we have $P \gg R$. Then, the quadratic problem (12) would be solved such that $\nu \ll \mu$. Therefore, selecting $P$ to be large enough would make the impact of $\nu$ negligible and this is desired because it is introduced only to create an additional degree of freedom for the control input that will aid the formulation of the control input to compensate for the disturbance rate induced by waves. In the control of ship roll motion, roll angle reduction is the main aim which implies $Q$ should be selected such that $q_{\phi}>q_{p}$. Then, the terminal weight $S$ can be obtained by solving the discrete-time algebraic Riccati equation (DARE); the importance of this will be discussed in Section 5 .

Remark 1. The cost function given in (12) would be minimised with respect to both $\mu_{k}$ and $\nu_{k}$. Consequently, the optimal solution of the cost function $J$ may not give $\mu_{k}^{*}$ that can be used to obtain the control signal as $u_{k}=\mu_{k}^{*}+u_{k-1}$ that would drive the system (9) to the desired state. Hence, it is necessary to devise a means to use both $\mu_{k}^{*}$ and $\nu_{k}^{*}$ to achieve the desired control. It is pertinent to emphasise here that we have no control over the actual system disturbance $\delta_{k}$, but $\nu_{k}$ is introduced to create two degree-of-freedom for the control that can be manipulated to help improve the rejection of the externally induced wave disturbance increment $\delta_{k}$.

The MPC problem described above is solved over a finite horizon as a deterministic optimal control problem using the current state as initial conditions and then the solution is implemented in a receding horizon manner. Thus, the feedback control law implemented, $u_{k}=u_{k-1}+\kappa_{N}$ takes $N$ future actions into account from the current state, and it is (implicitly) assumed that the influence of the actual decision on those beyond the horizon $N$ is appropriately summarised by a terminal cost. Hence, the use of a sufficiently large $N$ gives a reasonable approximation for an infinite horizon control problem.

Given the initial state $\tilde{x}_{0}$, we seek to obtain the control vectors

$$
\eta=\overbrace{\left[\mu_{t}^{T}, \mu_{t+1}^{T}, \ldots, \mu_{t+N-1}^{T}\right.}^{\bar{\mu}^{T}}, \overbrace{\nu_{t}^{T}, \nu_{t+1}^{T}, \ldots, \nu_{t+N-1}^{T}}^{T}]^{T},
$$


that minimise the optimisation problem (12). The optimal control problem is then solved at every sampling instant $t$ by using the current state as the initial conditions, that is, $\tilde{x}_{0}=\tilde{x}_{t}$. The solution of (12) reduces to the quadratic program:

$$
\eta^{*}=\underset{\eta}{\arg \min } \frac{1}{2} \eta^{T} H \eta+\eta^{T} F\left[\begin{array}{c}
\tilde{x}_{t} \\
\bar{r}
\end{array}\right]
$$

subject to: $\Gamma \eta \leq b$,

where

$$
H=\left[\begin{array}{cc}
\mathcal{G}^{T} \mathcal{Q G}+\mathcal{R} & \left(\mathcal{E}^{T} \mathcal{Q G}+\mathcal{V} \mathcal{G}\right)^{T} \\
\left(\mathcal{E}^{T} \mathcal{Q G}+\mathcal{V} \mathcal{G}\right) & \left(\mathcal{E}^{T} \mathcal{Q} \mathcal{E}+\mathcal{S}+\mathcal{V} \mathcal{E}+\mathcal{E}^{T} \mathcal{V}^{T}\right)
\end{array}\right], F^{T}=\left[\begin{array}{cc}
\mathcal{H}^{T} \mathcal{Q G} & \mathcal{H}^{T} \mathcal{Q E}+\mathcal{H}^{T} \mathcal{V}^{T} \\
-\mathcal{T} \mathcal{G} & -\mathcal{T} \mathcal{E}^{T}-\mathcal{U}
\end{array}\right]
$$

$$
\begin{gathered}
\mathcal{E}=\left[\begin{array}{cccc}
\tilde{B}_{d} & 0 & \cdots & 0 \\
\tilde{A} \tilde{B}_{d} & \tilde{B}_{d} & \cdots & 0 \\
\vdots & \vdots & \ddots & \vdots \\
\tilde{A}^{N-1} \tilde{B}_{d} & \tilde{A}^{N-2} \tilde{B}_{d} & \cdots & \tilde{B}_{d}
\end{array}\right], \mathcal{G}=\left[\begin{array}{cccc}
\tilde{B} & 0 & \cdots & 0 \\
\tilde{A} \tilde{B} & \tilde{B} & \cdots & 0 \\
\vdots & \vdots & \ddots & \vdots \\
\tilde{A}^{N-1} \tilde{B} & \tilde{A}^{N-2} \tilde{B} & \cdots & \tilde{B}
\end{array}\right], \\
\mathcal{H}=\left[\begin{array}{c}
\tilde{A} \\
\tilde{A}^{2} \\
\vdots \\
\tilde{A}^{N}
\end{array}\right], \mathcal{Q}=\left[\begin{array}{cccc}
\tilde{C}^{T} Q \tilde{C} & \cdots & 0 & 0 \\
\vdots & \ddots & \vdots & \vdots \\
0 & \cdots & \tilde{C}^{T} Q \tilde{C} & 0 \\
0 & \cdots & 0 & \tilde{C}^{T} S \tilde{C}
\end{array}\right], \mathcal{R}=\left[\begin{array}{ccc}
R & \cdots & 0 \\
\vdots & \ddots & \vdots \\
0 & \cdots & R
\end{array}\right],
\end{gathered}
$$

$$
\begin{gathered}
\mathcal{S}=\left[\begin{array}{cccc}
\tilde{D} Q \tilde{D}+P & \ldots & 0 \\
\vdots & \ddots & \vdots \\
0 & \cdots & \tilde{D} Q \tilde{D}+P
\end{array}\right], \mathcal{T}=\left[\begin{array}{cccc}
Q \tilde{C} & \cdots & 0 & 0 \\
\vdots & \ddots & \vdots & \vdots \\
0 & \cdots & Q \tilde{C} & 0 \\
0 & \cdots & 0 & S \tilde{C}
\end{array}\right], \\
\mathcal{U}=\left[\begin{array}{cccc}
Q \tilde{D} & \cdots & 0 & 0 \\
\vdots & \ddots & \vdots & \vdots \\
0 & \cdots & Q \tilde{D} & 0 \\
0 & \cdots & 0 & S \tilde{D}
\end{array}\right], \quad \mathcal{V}=\left[\begin{array}{cccc}
\tilde{D}^{T} Q \tilde{C} & \cdots & 0 & 0 \\
\vdots & \ddots & \vdots & \vdots \\
\vdots & \cdots & \tilde{D}^{T} Q \tilde{C} & 0 \\
0 & \cdots & 0 & \tilde{D}^{T} S \tilde{C}
\end{array}\right] .
\end{gathered}
$$

Also, $\bar{r}=\left[r_{t}^{T}, r_{t+1}^{T}, \ldots, r_{t+N-1}^{T}\right]^{T}$. The matrix $\Gamma$ and vector $b$ are used to implement the magnitude and rate constraint on the fin actuator which will be explicitly defined in the 
next subsection. The matrix, $H$ is an Hessian matrix and $F$ needs be computed online at every iteration as it contains terms such as $\tilde{x}_{t}$ that must be updated online.

It is convenient to obtain $\mu_{k}^{*}$ in every iteration from the computed optimal $\eta^{*}$, where only the first vector component of $\bar{\mu}^{*}$ is extracted based on the receding horizon principle. Similarly, to utilise the optimal disturbance $\nu_{k}^{*}$ along with $\mu_{k}^{*}$ (which is necessary to ensure that the plant is driven according to the minimisation control problem), we also extract the first vector component of $\bar{\nu}^{*}$. To enable an effective combination of the optimal values $\mu_{k}^{*}$ and $\nu_{k}^{*}$, we define the control signal increment that is desired in every time step $k$ as

$$
\psi_{k} \triangleq \mu_{k}^{*}+\lambda_{k}
$$

Here, $\lambda_{k}$ represents a component of the control signal increment $\psi_{k}$ that is dependent on $\nu_{k}^{*}$ and gives an additional freedom of control. Hence, the controlled augmented velocity model (10) is then given as

$$
\tilde{x}_{k+1}=\tilde{A} \tilde{x}_{k}+\tilde{B} \psi_{k}+\tilde{B}_{d} \delta_{k}
$$

To incorporate $\nu_{k}^{*}$ into the control while ensuring that the output error $e_{k}$ is minimised and the effects of the disturbance increment $\delta_{k}$ is reduced, we need to ensure that

$$
\tilde{B} \lambda_{k}=B_{d}\left(\nu_{k}^{*}-\hat{\delta}_{k}\right)
$$

$\forall k \geq 0$. Note that $\hat{\delta}_{k}$ can be obtained either by measurement or estimation of the disturbance signal $d_{k}^{w}$. Furthermore, it can be seen from (19) that the estimated disturbance increment $\hat{\delta}_{k}$ is in direct opposition to the actual system disturbance rate $\delta_{k}$ in (18). Since the induced wave disturbances cannot be measured in the roll stabilisation control problem, it is important to use a good estimate of the actual disturbance in order to ensure that the effects of the disturbance increment is adequately reduced. In general, $\tilde{B}$ is not invertible; hence, we obtain $\lambda_{k}$ as

$$
\lambda_{k}=\left(\tilde{B}^{T} \tilde{B}\right)^{-1} \tilde{B}^{T} \tilde{B}_{d}\left(\nu_{k}^{*}-\hat{\delta}_{k}\right)
$$

Therefore, we are set to define the optimal control signal $u_{k}^{*}$ to be applied to the fin via its actuator in every time step as

$$
u_{k}^{*}=u_{k-1}+\mu_{k}^{*}+\lambda_{k} .
$$


Remark 2. In general, $\left(\tilde{B}^{T} \tilde{B}\right)$ is almost always non-singular because $\tilde{B}$ is a tall matrix having $(n+p)$ rows and $m$ columns, where $(n+p)>m$. And in a well defined system, $\operatorname{rank}(\tilde{B})=m$ so that $\tilde{B}^{T} \tilde{B}$ is usually an $m \times m$ matrix with $\operatorname{rank}\left(\tilde{B}^{T} \tilde{B}\right)=m$, which means that an inverse will always exist. However, if the inverse operation is not applicable, one can use the pseudo inverse of the matrix.

\subsection{Constraints handling}

In this subsection, the formulation of the input and input rate constraint on the actuator fin are presented.

\subsubsection{Input constraint}

Let the upper and lower limit of the actuator fin input angle be given as,

$$
u_{\min } \leq u_{k} \leq u_{\max } \quad \forall k \in\{t, \ldots, t+N-1\}
$$

Recall that the control signal at any sampling instant $t$ is given by

$$
u_{t}=\mu_{t}+\lambda_{t}+u_{t-1}
$$

By defining $\Lambda \triangleq\left(\tilde{B}^{T} \tilde{B}\right)^{-1} \tilde{B}^{T} \tilde{B}_{d}$, one can write $(20)$ as

$$
\lambda_{t}=\Lambda \nu_{t}^{*}-\Lambda \hat{\delta}_{t}
$$

Thus, one can readily formulate the control input constraint in the form of the inequality:

$$
\overbrace{\left[\begin{array}{cc}
\mathcal{L}_{1} & \mathcal{L}_{2} \\
-\mathcal{L}_{1} & -\mathcal{L}_{2}
\end{array}\right]}^{\Gamma_{1}} \eta \overbrace{\left[\begin{array}{c}
\bar{u}_{\max } \\
-\bar{u}_{\text {min }}
\end{array}\right]-\left[\begin{array}{c}
\tilde{I}_{n_{u}} \\
-\tilde{I}_{n_{u}}
\end{array}\right] u_{t-1}+\left[\begin{array}{c}
\tilde{I}_{n_{u}} \\
-\tilde{I}_{n_{u}}
\end{array}\right] \Lambda \hat{\delta}_{t},}^{b_{1}},
$$

where

$$
\begin{gathered}
\mathcal{L}_{1}=\left[\begin{array}{cccc}
I & 0 & \cdots & 0 \\
I & I & \cdots & 0 \\
\vdots & \vdots & \ddots & \vdots \\
I & I & \cdots & I
\end{array}\right], \mathcal{L}_{2}=\left[\begin{array}{cccc}
\Lambda & 0 & \cdots & 0 \\
\Lambda & \Lambda & \cdots & 0 \\
\vdots & \vdots & \ddots & \vdots \\
\Lambda & \Lambda & \cdots & \Lambda
\end{array}\right] \tilde{I}_{n_{u}}=\left[\begin{array}{c}
I \\
I \\
\vdots \\
I
\end{array}\right] . \\
\bar{u}_{\min }=\left[\begin{array}{c}
u_{\min } \\
\vdots \\
u_{\min }
\end{array}\right] \text { and } \bar{u}_{\max }=\left[\begin{array}{c}
u_{\max } \\
\vdots \\
u_{\max }
\end{array}\right] .
\end{gathered}
$$




\subsubsection{Input rate constraint}

The constraint on the fin actuator rate must be implemented on $\psi_{k}$, which is now the actual control input increment (and not $\mu_{k}$ ) due to the introduction of $\lambda_{k}$. Let the constraint be given as

$$
\psi_{\min } \leq \psi_{k} \leq \psi_{\max } \quad \forall k \in\{t, \ldots, t+N-1\} .
$$

where $\psi_{\min }$ is the minimum fin angle rate and $\psi_{\max }$ is the maximum angular velocity. The input rate constraint can be formulated to obtain:

$$
\overbrace{\left[\begin{array}{cc}
\mathcal{L}_{3} & \mathcal{L}_{4} \\
-\mathcal{L}_{3} & -\mathcal{L}_{4}
\end{array}\right]}^{\Gamma_{2}}\left[\begin{array}{l}
\bar{\mu} \\
\bar{\nu}
\end{array}\right] \leq \overbrace{\left[\begin{array}{c}
\bar{\psi}_{\max } \\
-\bar{\psi}_{\min }
\end{array}\right]+\left[\begin{array}{c}
\tilde{I}_{n_{u}} \\
-\tilde{I}_{n_{u}}
\end{array}\right] \Lambda \hat{\delta}_{t}}^{b_{2}}
$$

where $L_{3}=\operatorname{blkdiag}(I, \ldots, I)$ and $L_{4}=\operatorname{blkdiag}(\Lambda, \ldots, \Lambda)$,

$$
\bar{\psi}_{\min }=\left[\begin{array}{c}
\psi_{\min } \\
\vdots \\
\psi_{\min }
\end{array}\right], \bar{\psi}_{\max }=\left[\begin{array}{c}
\psi_{\max } \\
\vdots \\
\psi_{\max }
\end{array}\right] .
$$

where blkdiag $(X, \ldots, X)$ represents a diagonal matrix with diagonal entries of $X$. Therefore, the matrix $\Gamma$, and vector $b$ which are required to implement the inequality constraint on the optimisation problem are given as follows: $\Gamma=\left[\begin{array}{c}\Gamma_{1} \\ \Gamma_{2}\end{array}\right], b=\left[\begin{array}{l}b_{1} \\ b_{2}\end{array}\right]$. The disturbance observerbased MPC described in this section can be implemented by performing the following steps at each iteration:

1. Initialise the states by taking the initial measurements $x_{k}=x_{0}$ and obtain the estimate of disturbance increment $\hat{\delta}_{k}$.

2. Solve the constrained optimisation problem (12) and then obtain $\mu_{k}^{*}$ and $\nu_{k}^{*}$ as the first elements in $\bar{\mu}^{*}$ and $\bar{\nu}^{*}$, respectively.

3. Compute $\lambda_{k}$ using definition (20).

4. Determine the current control signal $u_{k}^{*}$ according to (21) and apply it to the plant (1).

5. Set $k \leftarrow k+1$ and return to step 1 .

\section{Parameter estimation}

The proposed MPC algorithm in the previous section assumes that the estimate of the wave-induced disturbances is available in each time step. Since the sea state and sailing 
conditions of the vessel change with time, the estimate needs to be updated in every sampling instant $k$. Consider the discrete-time plant (9) along with $\zeta_{k}$, the signal to be estimated:

$$
\begin{aligned}
x_{k+1} & =A x_{k}+B u_{k}+B d_{d} w, \\
y_{k} & =C x_{k}+D d_{k}, \\
\zeta_{k} & =E x_{k}+F d_{k} .
\end{aligned}
$$

Since there are no measured disturbances and all system states are measurable, $C=I_{2}$ and $D=0$. To characterise the input wave disturbances to obtain a good estimate, we propose a frequency shaping filter whose dynamics are given by

$$
\begin{gathered}
v_{k+1}=A_{i} v_{k}+B_{i} w_{k}, \\
d_{k}=C_{i} v_{k}+D_{i} w_{k} .
\end{gathered}
$$

Generally, the dynamic filter can be unstable, which implies that the eigenvalues of $A_{i}$ can be on or outside the unit circle.

Remark 3. The signal $\zeta_{k}$ is constructed such that it collects all the signals that need to be estimated. For instance, if all system states and the input disturbances need to be estimated, $\zeta_{k}$ will be constructed as

$$
\zeta_{k}=\left[\begin{array}{l}
I \\
0
\end{array}\right] x_{k}+\left[\begin{array}{l}
0 \\
I
\end{array}\right] d_{k} .
$$

It is important to note that the estimation of all system states and input disturbance would likely lead to poor performance unless the disturbances are characterised properly. Indeed, the frequency shaping filter is a tool to properly characterise the disturbance signal. The filter can be used to characterise the disturbance vector in a number of ways. It would usually be convenient to include a low-pass or band-pass component in the input filter, whose bandwidth is to be decided based on the knowledge about the disturbance signals. Alternatively, one might use such a filter at the output to generate a $\zeta$ signal that in fact represents the component of the signal to be estimated in the frequency band of interest (rather than the signal as it is).

The aim of the design of the estimator is to effectively utilise the measurement vector $y_{k}$ to obtain reliable estimates of $\zeta_{k}$. This dynamic estimator is of the form

$$
\begin{aligned}
\xi_{k+1} & =A_{e} \xi_{k}+B_{e} y_{k}, \\
\hat{\zeta}_{k} & =C_{e} \xi_{k}+D_{e} y_{k},
\end{aligned}
$$


where $\hat{\zeta} \in \mathbb{R}^{n_{e}}$ denotes the estimates of $\zeta_{k}$ and $A_{e}, B_{e}, C_{e}$ and $D_{e}$ are the observer matrices to be found in order to realise the estimator.

The estimator is to be designed in a way to minimise the prediction error in some appropriate norm. It is more convenient to use a scaled version of the error signal as

$$
\varepsilon_{k}=W\left(\zeta_{k}-\hat{\zeta}_{k}\right)
$$

where $W$ is the weighting matrix typically taken to be of a diagonal form. It enables the adjustment of the relative emphasis on the components of the error signal.

Based on the above, the estimator design problem can now be stated as follows: given the closed-loop plant (28) and the frequency shaping filter (29), the aim is to design an estimator (31) such that the transfer matrix $\mathcal{T}_{\varepsilon w}$ from the input $w$ of the filter (29) to the scaled error signal $\varepsilon$ in (32) is stable and has an upper bound $\gamma$ defined in some appropriate norm.

Remark 4. It is interesting to observe that the disturbance frequency shaping filter can be introduced artificially to the problem (and more conveniently so in a discrete-time setting) even when it is not considered in the original problem formulation. Consider the discrete plant (28) for which the aim is to design an estimator (31). Since no exo-system exists because $A_{i}, B_{i}, C_{i}$ are all void and $D_{i}=I$, we would typically consider minimising a particular norm of the transfer matrix $\mathcal{T}_{\varepsilon d}$ from $d^{w}$ to $\varepsilon$. If the disturbances have dominant time-varying components, it might be preferable to use a stable filter whose bandwidth is chosen in a way to appropriately characterise the disturbances.

\subsection{Problem solution}

In this note, an observer-based solution to the estimation problem (defined in the previous section) with disturbance frequency shaping filter is presented. To implement the observer based solution, the plant (28) dynamics are merged with that of the frequency shaping filter 
(29). In this fashion, we express the dynamics of the extended plant as follows:

$$
\begin{aligned}
\underbrace{\left[\begin{array}{c}
x_{k+1} \\
v_{k+1}
\end{array}\right]}_{\vartheta_{k+1}} & =\underbrace{\left[\begin{array}{cc}
A & B_{d} C_{i} \\
0 & A_{i}
\end{array}\right]}_{A_{o}} \underbrace{\left[\begin{array}{c}
x_{k} \\
v_{k}
\end{array}\right]}_{\vartheta_{k}}+\underbrace{\left[\begin{array}{c}
B \\
0
\end{array}\right]}_{B_{o}} u_{k}+\underbrace{\left[\begin{array}{c}
B_{d} D_{i} \\
B_{i}
\end{array}\right]}_{\bar{B}_{o}} w_{k}, \\
y_{k} & =\underbrace{\left[\begin{array}{ll}
C_{y} & D_{y} C_{i}
\end{array}\right]}_{C_{o}} \vartheta_{k}+\underbrace{D_{y} D_{i}}_{D_{o}} w_{k}, \\
\zeta_{k} & =\underbrace{\left[\begin{array}{cc}
E & F C_{i}
\end{array}\right]}_{E_{o}} \vartheta_{k}+\underbrace{F D_{i}}_{F_{o}} w_{k} .
\end{aligned}
$$

The observer to estimate the state and the output signals of the extended plant model (33) can be constructed as,

$$
\begin{aligned}
\hat{\vartheta}_{k+1} & =A_{o} \hat{\vartheta}_{k}+B_{o} u_{k}-L\left(y_{k}-\hat{y}_{k}\right), \\
\hat{y}_{k} & =C_{o} \hat{\vartheta}_{k}, \\
\hat{\zeta}_{k} & =E_{o} \hat{\vartheta}_{k} .
\end{aligned}
$$

We can establish the detectability condition of the augmented observer based on the results given in Pannocchia and Rawlings (2003). The detectability of the augmented system (33) is guaranteed provided that the plant $(A, B, C)$ is detectable and the condition

$$
\operatorname{rank}\left[\begin{array}{cc}
I-A & -B_{d} C_{i} \\
C_{y} & D_{y} C_{i}
\end{array}\right]=n_{d}+n_{x},
$$

also holds and this is necessary and sufficient to guarantee the existence of a stable estimator.

Remark 5. The augmentated system (33) and condition (35) reduce to those given in Pannocchia and Rawlings (2003) by using a frequency shaping filter where $A_{i}, B_{i}$ and $C_{i}$ are all identity matrices and $D_{i}=0$. Therefore, the augmentation and detectability condition given in Pannocchia and Rawlings (2003) are particular cases of the more general representations given by (33) and (35).

We note here that the matrix $L$ is the observer gain matrix to be computed. We also emphasise at this point that the observer-based estimator corresponds to the choice of the realisation matrices in a specific way as follows:

$$
\left[\begin{array}{cc}
A_{e} & B_{e} \\
C_{e} & D_{e}
\end{array}\right]=\left[\begin{array}{cc}
A_{o}+L C_{o} & -L \\
E_{o} & 0
\end{array}\right] .
$$


The state estimation error is computed as $\epsilon_{k} \triangleq \vartheta_{k}-\hat{\vartheta}_{k}$. Therefore, the evolution of $\epsilon$ and $\varepsilon$ are given as,

$$
\begin{aligned}
\epsilon_{k+1} & =\underbrace{\left(A_{o}+L C_{o}\right)}_{\mathcal{A}} \epsilon_{k}+\underbrace{\left(B_{o}+L D_{o}\right)}_{\mathcal{B}} w_{k}, \\
\varepsilon_{k} & =\underbrace{W E_{o}}_{\mathcal{C}} \epsilon_{k}+\underbrace{W F_{0}}_{\mathcal{D}} w_{k} .
\end{aligned}
$$

Based on this representation of the error dynamics, which is basically a realisation of $\mathcal{T}_{\varepsilon w}$, one can easily arrive at LMI conditions that ensure the stability of a specified norm bound on $\mathcal{T}_{\varepsilon w}$.

\section{2. $\mathcal{H}_{2}$ Synthesis}

The matrix inequality conditions for $\left\|\mathcal{T}_{\varepsilon w}\right\|_{2}<\gamma$ can be expressed (Scherer and Weiland, 2015) in discrete-time as follows:

$$
\begin{gathered}
\operatorname{tr}(Z)<\gamma, \\
{\left[\begin{array}{ccc}
\mathcal{X} & 0 & \star \\
0 & \gamma I & \star \\
\mathcal{X} \mathcal{A} & \mathcal{X} \mathcal{B} & X
\end{array}\right] \succ 0,\left[\begin{array}{ccc}
\mathcal{X} & 0 & \mathcal{C}^{T} \\
0 & \gamma I & \mathcal{D}^{T} \\
\mathcal{C} & \mathcal{D} & Z
\end{array}\right] \succ 0 .}
\end{gathered}
$$

By introducing $M \triangleq X L$, we can obtain an LMI condition with $\mathcal{X}=X$ as follows:

$$
\begin{gathered}
\operatorname{tr}(Z)<\gamma, \quad\left[\begin{array}{ccc}
X & \star & \star \\
0 & \gamma I & \star \\
W E_{o} & W F_{o} & Z
\end{array}\right] \succ 0, \\
{\left[\begin{array}{ccc}
X & 0 & \star \\
0 & \gamma I & \star \\
X A_{o}+M C_{o} & X B_{o}+M D_{o} & X
\end{array}\right] \succ 0 .}
\end{gathered}
$$

Then, the observer gain can be computed from a solution of this LMI problem as $L=$ $X^{-1} M$ for the minimum achievable $\gamma$ which satisfies (39).

By obtaining the gain $L$, the observer (34) can conveniently be implemented to estimate disturbances induced by waves which complete the design of the observer-based MPC proposed in this note. The functional block diagram of the proposed controller is depicted in 


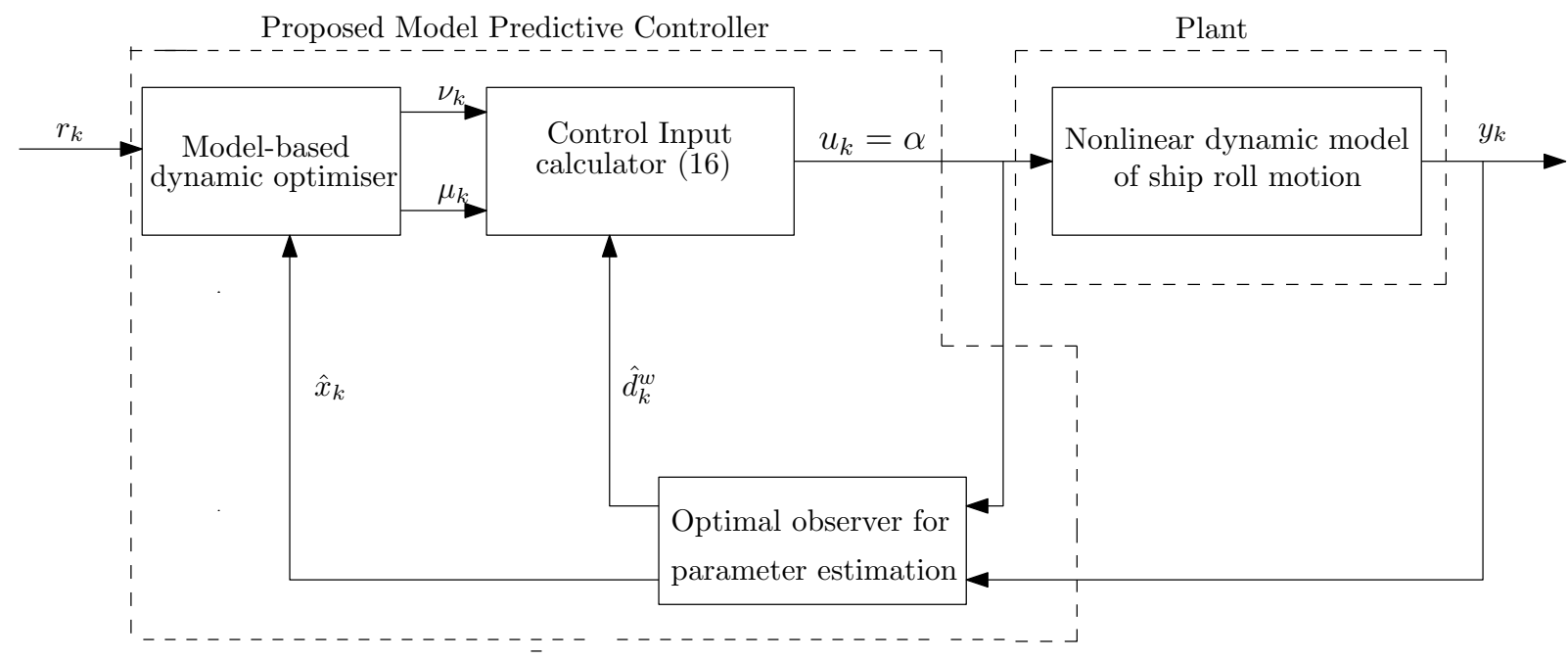

Figure 1: Block diagram representation of the proposed MPC scheme with combined state and disturbance estimator for roll motion stabilisation via fin control.

Figure 1, where the outputs of a model-based optimiser are the 'optimal disturbance' increment $\nu_{k}$, and optimal control increment $\mu_{k}$. Based on the current output of the plant and the applied control signal, the optimal observer provides an estimate for the states and disturbances. Unlike the estimated states that are fed back into the model-based optimiser, the disturbance estimates are fed into the control input calculator which gives a control signal $u$ that is a function of the two outputs from the optimiser and the (increment) disturbance estimates as in (21).

\section{Stability Analysis}

This section presents an analysis of the stability of the proposed disturbance observerbased MPC with increment disturbance compensator. Considering the uncertain nature of the ship roll dynamics, it is essential to show that the proposed MPC is robustly stable. In MPC framework, feasibility and stability analysis can be performed by showing that the optimisation problem is recursively feasible and the cost function is a Lyapunov function. In carrying out this analysis, two approaches are widely used. The first considers an additional terminal constraint $\tilde{x}_{t+N}=0$ while the second method defines $\tilde{x}_{t+N} \in X_{f}$ where $X_{f}$ is a convex set. It is well known that the former approach reduces the size of the feasibility region. Hence, we will adopt the second approach to increase the region of attraction. 


\subsection{Nominal Stability Analysis}

In this subsection, we will discuss the feasibility and stability of the proposed controller under nominal plant condition. For convenience, we assume that the disturbance $d_{k}^{w}$ is either zero or constant which implies that $\hat{\delta}_{k}=\delta_{k}=0$.

Definition 5.1. A set $\mathcal{O}$ is said to be positively invariant for system $\tilde{x}_{k+1}=g\left(\tilde{x}_{k}, \kappa_{N}\left(\tilde{x}_{k}\right)\right)$ if $\tilde{x}_{0} \in \mathcal{O} \Longrightarrow \tilde{x}_{k} \in \mathcal{O} \forall k \in \mathbb{N}_{+}$.

Assumption 1. The terminal set $X_{f}$ is invariant under the local control law $\kappa_{N}\left(\tilde{x}_{k}\right)$ which implies $\tilde{x}_{k+1}=\tilde{A} \tilde{x}_{k}+\mathrm{B} \kappa_{N}\left(\tilde{x}_{k}\right) \in X_{f} \forall \tilde{x}_{k} \in X_{f}$; where $\mathrm{B}=\left[\begin{array}{ll}\tilde{B} & \tilde{B}_{d}\end{array}\right]$. The state and input constraints on $\mathcal{X}_{f}$ are fulfilled: $\mathcal{X}_{f} \subseteq \mathcal{X}, \kappa_{N}\left(\tilde{x}_{k}\right) \in \mathcal{U} \forall \tilde{x}_{k} \in \mathcal{X}_{f}$.

Proposition 5.2. Under Assumption 1, a predictive control law $\kappa_{N}: \mathbb{R}^{n_{x}} \mapsto \mathbb{R}^{n_{u}}$ defined for the nominal plant $g\left(\tilde{x}_{k}, \mu_{k}, \nu_{k}\right)=\tilde{A} \tilde{x}_{t+k}+\tilde{B} \mu_{k}^{*}+\tilde{B}_{d} \nu_{k}^{*}$ by minimising the cost function (12) subject to the constraint $\tilde{x}_{t+N} \in X_{f}$ is recursively feasible provided that the initial condition of the state $\tilde{x}_{t}$ is feasible and the receding horizon principle is applied.

Proof. First, let us express the cost function (12) in a manner that readily shows its similarity to the conventional quadratic MPC cost function and constraints as follows:

$$
\mathbb{Q}(\tilde{x}): \min _{\overline{\mathrm{u}}} \underbrace{\frac{1}{2} e_{t+N}^{T} S e_{t+N}}_{\text {Terminal cost }}+\underbrace{\frac{1}{2} \sum_{k=0}^{N-1}\left\{e_{t+k}^{T} Q e_{t+k}+\mathbf{u}_{t+k}^{T} \mathrm{Ru}_{t+k}\right\}}_{\text {Stage cost }},
$$

subject to:

$$
\begin{gathered}
\mathrm{u}_{t+k} \in \mathcal{U}, \\
\tilde{x}_{t+k} \in X, \\
\tilde{x}_{t+N} \in X_{f} .
\end{gathered}
$$

where $\mathbf{u}_{t+k}=\left[\begin{array}{c}\mu_{t+k}^{*} \\ \delta_{t+k}^{*}\end{array}\right]$ is obtained by solving the MPC minimisation problem, $\mathrm{R}=\left[\begin{array}{cc}R & 0 \\ 0 & P\end{array}\right]$ and $\mathcal{U}$ and $\mathcal{X}$ are convex sets. According to (21), the control input to be implemented at any time $k$ would then be $u_{k}^{*}=[I \Lambda] \mathbf{u}_{k}+u_{k-1}$. Based on the cost function given in (40), it is evident that the proposed MPC cost function is essentially the same as that used in standard MPC problem. However, this work has divided the control $\mathbf{u}_{k}$ into two components to enable the utilisation of an optimal disturbance. Based on the cost (40), the nominal plant can be 
re-written as $g\left(\tilde{x}_{k}, \mathbf{u}_{k}\right)=\tilde{A} \tilde{x}_{t+k}+\mathrm{Bu}_{k}$. If the system is driven by a feedback control law $\kappa_{N}: \mathbb{R}^{n_{x}} \mapsto \mathbb{R}^{n_{u}}$, the solution of the closed-loop system is $g\left(\tilde{x}_{k}, \kappa_{N}\left(\tilde{x}_{k}\right)\right)$.

Since the initial state condition $\tilde{x}_{t}$ is assumed to be feasible, the solution to the QP (40), as a function of the state, yields the control sequence $\overline{\mathbf{u}}(\tilde{x})=\left\{\mathbf{u}_{t}(\tilde{x}), \mathbf{u}_{t+1}(\tilde{x}), \ldots\right.$, $\left.\mathbf{u}_{t+N-1}(\tilde{x})\right\}$ and the corresponding state trajectory is $\left\{\tilde{x}_{t}, \tilde{x}_{t+1}, \ldots, \tilde{x}_{t+N}\right\}$. At the next time step, the state becomes $\tilde{x}_{t+1}$ and the corresponding optimal control sequence $\overline{\mathrm{u}}(\tilde{x})=$ $\left\{\mathbf{u}_{t+1}(\tilde{x}), \mathbf{u}_{t+2}(\tilde{x}), \ldots, \kappa_{N}\left(\tilde{x}_{t+N}\right)\right\}$ is feasible because (i) $x_{t+N} \in X_{f} \rightarrow \kappa_{N}\left(\tilde{x}_{t+N}\right)$ is feasible and (ii) $\tilde{x}_{t+N+1}=\tilde{A} \tilde{x}_{t+N}+\mathrm{B} \kappa_{N}\left(\tilde{x}_{t+N}\right) \in X_{f}$. Therefore, recursive feasibility is implied by terminal cost.

To show that the predictive control law is asymptotically stable, we make the following additional assumptions.

Assumption 2. The stage cost is strictly positive and only zero at the origin which is assumed to be $\tilde{x}=0$ and $\mathbf{u}=0$.

Assumption 3. In the terminal set $X_{f}$, the terminal cost is a continuous Lyapunov function and it satisfies: $\ell_{1}\left(\tilde{x}_{k+1}\right)-\ell_{1}\left(\tilde{x}_{k}\right) \leq-\ell_{2}\left(\tilde{x}_{k}, \kappa_{N}\left(\tilde{x}_{k}\right)\right) \forall \tilde{x}_{k} \in X_{f}$.

Theorem 5.3. Under Assumption 1, 2 and 3, a predictive control law $\kappa_{N}\left(\tilde{x}_{k}\right)$ for the nominal plant $g\left(\tilde{x}_{k}, \mathbf{u}_{k}\right)$ based on the minimisation of the cost (40) is asymptotically stable.

Proof. Consider the alternative form of the cost (12) given in (40), one can conveniently re-write the cost function as

$$
J\left(\tilde{x}_{t}\right)=\ell_{1}\left(\tilde{x}_{t+N}\right)+\sum_{k=0}^{N-1} \ell_{2}\left(\tilde{x}_{t+k}, \mathrm{u}_{\mathrm{t}+\mathrm{k}}\right),
$$

where $\ell_{1}\left(x_{t+N}\right)=e_{t+N}^{T} S e_{t+N}$ and $\ell_{2}\left(\tilde{x}_{t+k}, \mathbf{u}_{\mathrm{t}+\mathrm{k}}\right)=e_{t+k}^{T} Q e_{t+k}+\mu_{t+k}^{T} R \mu_{t+k}+\delta_{t+k}^{T} P \delta_{t+k}$. The feasible input sequence $\overline{\mathbf{u}}(\tilde{x})=\left\{\mathbf{u}_{t}(\tilde{x}), \mathbf{u}_{t+1}(\tilde{x}), \ldots, \kappa_{N}\left(\tilde{x}_{t+N}\right)\right\}$ corresponds to the state $\tilde{x}_{t+1}$ 
and the cost function corresponding to this state is given as

$$
\begin{aligned}
& J\left(\tilde{x}_{t+1}\right) \leq \ell_{1}\left(\tilde{x}_{t+N+1}\right)+\sum_{k=1}^{N} \ell_{2}\left(\tilde{x}_{t+k}, \mathbf{u}_{\mathbf{t}+\mathrm{k}}\right) \\
&= \ell_{1}\left(\tilde{A} \tilde{x}_{t+N}+\mathrm{B} \kappa_{N}\left(\tilde{x}_{t+N}\right)\right)+\sum_{k=0}^{N-1} \ell_{2}\left(\tilde{x}_{t+k}, \mathbf{u}_{\mathbf{t}+\mathrm{k}}\right) \\
& \quad-\ell_{2}\left(\tilde{x}_{t}, \mathbf{u}_{\mathrm{t}}\right)+\ell_{2}\left(\tilde{x}_{t+N}, \mathbf{u}_{\mathbf{t}+\mathrm{N}}\right) \\
&=J\left(\tilde{x}_{t}\right)-\ell_{2}\left(\tilde{x}_{t}, \mathrm{u}_{\mathbf{t}}\right)+p(\tilde{x}), \\
& \Longrightarrow J\left(\tilde{x}_{t+1}\right)-J\left(\tilde{x}_{t}\right) \leq-\ell_{2}\left(\tilde{x}_{t}, \mathbf{u}_{\mathbf{t}}\right)+p(\tilde{x})
\end{aligned}
$$

where $p(\tilde{x})=\ell_{1}\left(\tilde{A} \tilde{x}_{t+N}+\mathrm{B} \kappa_{N}\left(\tilde{x}_{t+N}\right)\right)-\ell_{1}\left(\tilde{x}_{t+N}\right)+\ell_{2}\left(\tilde{x}_{t+N}, \kappa_{N}\left(\tilde{x}_{t+N}\right)\right)$ and from Assumption $3, p(\tilde{x}) \leq 0$. Since $\ell_{2}\left(\tilde{x}_{t}, \mathrm{u}_{\mathrm{t}}\right)$ is positive definite based on Assumption 2, we have $J\left(\tilde{x}_{t+1}\right) \leq$ $J\left(\tilde{x}_{t}\right)$. This completes the proof since $J\left(\tilde{x}_{t+k}\right)$ is a Lyapunov function decreasing along the closed-loop trajectories.

Since the asymptotic stability and recursive feasibility proofs above rely on Assumptions 1, 2 and 3, we will now show that the three assumptions are valid.

Let $\kappa_{N}\left(\tilde{x}_{k}\right)=\mathrm{K} \tilde{x}_{k}$ for all $\tilde{x}_{k} \in X_{f}$ where $X_{f} \subseteq X$. Note that this control law is only valid at the $N^{\text {th }}$ step or when the state trajectory enters the terminal set $x_{f}$.

Claim 5.4. The terminal set $x_{f}$ is invariant under the local control law $\kappa_{N}\left(\tilde{x}_{k}\right)=\mathrm{K} \tilde{x}_{k}$ if the gain $\mathrm{K}$ is computed as the solution to the DARE:

$$
S_{\infty}-(\tilde{A}+\mathrm{BK})^{T} S_{\infty}(\tilde{A}+\mathrm{BK})=Q+F_{\infty}^{T} R \mathrm{~K} .
$$

Proof. Choose the terminal weight $S=S_{\infty}$. Also choose the terminal set $x_{f}$ to be a maximally invariant set for the closed-loop system $\tilde{x}_{k+1}=(\tilde{A}+\mathrm{BK}) \tilde{x}_{k}$. Then, $\tilde{x}_{k+1}=$ $(\tilde{A}+\mathrm{BK}) \tilde{x}_{k} \in X_{f}$ for all $x_{k} \in X_{f}$. Hence, Assumption 1 is satisfied.

Since $\ell_{2}$ is positive definite, Assumption 2 is fulfilled. Indeed Assumption 3 represents optimal cost decrease which is shown to be satisfied by the following Lemma.

Lemma 5.5. For all $\tilde{x} \in X_{f}$, the optimal cost decrease: $\ell_{1}\left(\tilde{x}_{k+1}\right)-\ell_{1}\left(\tilde{x}_{k}\right) \leq-\ell_{2}\left(\tilde{x}_{k}, \kappa_{N}\left(\tilde{x}_{k}\right)\right) \forall$ $\tilde{x}_{k} \in X_{f}$, holds.

Proof. Based on the definition of $\ell_{1}\left(\tilde{x}_{k}\right)$, the left hand side (LHS) of the optimal cost decrease inequality can be written as $\tilde{x}_{k+1}^{T} S \tilde{x}_{k+1}-\tilde{x}_{k}^{T} S \tilde{x}_{k}$. Since $\tilde{x}_{k+1}=(\tilde{A}+\mathrm{BK}) \tilde{x}_{k}$, the 
LHS becomes $\tilde{x}_{k}^{T}(\tilde{A}+\mathrm{BK})^{T} S_{\infty}(\tilde{A}+\mathrm{BK}) \tilde{x}_{k}-\tilde{x}_{k}^{T} S_{\infty} \tilde{x}_{k}$. On the basis of (44), we have

$$
\begin{array}{r}
\tilde{x}_{k}^{T}(\tilde{A}+\mathrm{BK})^{T} S_{\infty}(\tilde{A}+\mathrm{BK}) \tilde{x}_{k}-\tilde{x}_{k}^{T} S_{\infty} \tilde{x}_{k} \\
=-\tilde{x}_{k}^{T}\left(Q+F_{\infty}^{T} R \mathrm{~K}\right) \tilde{x}_{k},
\end{array}
$$

Recall that $Q \geq 0$ and $R>0$ which implies that $\tilde{x}_{k}^{T}\left(Q+F_{\infty}^{T} R \mathrm{~K}\right) \tilde{x}_{k} \geq 0$; thus, the LHS of (45) is strictly negative and only zero at the origin.

\subsection{Robust Stability Analysis}

This subsection aims to extend the results from the nominal case to the uncertain plant which means that $d_{k}^{w}$ is varying and uncertain such that $\delta_{k}$ is non-zero. This scenario applies to the ship roll motion stabilisation problem which is the focus of this paper. Hence, we shall show that the proposed algorithm is inherently robust to the wave induced varying and uncertain disturbances $d_{k}^{w}$. To proceed, let (18) be written more explicitly as

$$
\tilde{x}_{k+1}=\tilde{A} \tilde{x}_{k}+\tilde{B}\left(\mu_{k}+\lambda_{k}\right)+\tilde{B}_{d} \delta_{k}
$$

By substituting (20) into (46), the following equation can be obtained:

$$
\tilde{x}_{k+1}=\tilde{A} \tilde{x}_{k}+\overbrace{\left[\begin{array}{ll}
\tilde{B} & \tilde{B}_{d}
\end{array}\right]}^{\mathrm{B}} \mathrm{u}_{k}+\overbrace{\tilde{B}_{d}\left(\delta_{k}-\hat{\delta}_{k}\right)}^{\mathrm{w}} .
$$

From (47), the formulated state-space model is of the form of an uncertain system with input disturbance $\mathbf{w}$. The perturbed system is modelled as the difference equation:

$$
G\left(\tilde{x}_{k}\right)=\left\{g\left(\tilde{x}_{k}, \kappa_{N}\left(\tilde{x}_{k}+e_{k}\right)\right)+\mathrm{w} \mid e_{k} \in \mathbb{E}, \mathbf{w} \in \mathbb{W}\right\}
$$

where $e_{k}$ represent the error in the state because it is not precisely known due to measurement and/or estimation error and the generic solution of the perturbed system is denoted $\phi_{k}^{\mathrm{ew}}(\tilde{x})$.

Remark 6. In general, the wave induced uncertainty $\mathrm{w}$ will be a small parameter because it represents a mitigated increment of the wave disturbance between two time steps and is zero when the magnitude of the estimated disturbance increment $\hat{\delta}_{k}$ equals the magnitude of the actual system disturbance increment $\delta_{k}$. Therefore, the additive wave motion dependent disturbance $\mathrm{w} \in \mathbb{W}$ is compact and bounded, and this holds even for rapidly changing waveinduced disturbances provided that the estimate $\hat{\delta}$ of the actual disturbance increment $\delta_{k}$ can be obtained. 
Proposition 5.6 (Grimm et al. (2004)). Consider the nominal closed-loop system $g\left(\tilde{x}_{k}, \kappa_{N}(\tilde{x})\right)$. For any $\epsilon_{p}>0$, there exist $\delta_{p}>0$ such that if $\{\mathbb{W}, \mathbb{E}\} \in \delta_{p} \mathbb{B}$, the robust asymptotic stability condition: $\phi_{k}^{\mathrm{ew}}(\tilde{x}) \leq \beta(|\tilde{x}|, k)+\epsilon_{p}$ holds for $G\left(\tilde{x}_{k}\right)$ provided that: (1) a continuous Lyapunov function exists for $g\left(\tilde{x}_{k}, \kappa_{N}(\tilde{x})\right)$. (2) $\mathbb{Q}(\tilde{x})$ is recursively feasible.

Note that $\mathbb{B}$ is a unit ball and $\beta$ is of class $\mathcal{K} \mathcal{L}$ function. Therefore, the method in this paper provides robust asymptotic stability since the conditions given in Proposition 5.6 have been shown to hold in the previous subsection.

Remark 7. The proposed method is a re-arranged version of 'standard' uncertain systems with measurement/estimation error and additive input disturbance. The analysis shows that the proposed method falls within the robust asymptotic stability proofs which can be found in (Grimm et al., 2004; Pannocchia et al., 2011a,b). The benefit of the proposed reformulation is that it makes it possible to compensate for both the magnitude and rate of the input disturbance.

\section{Numerical simulation}

A numerical simulation is conducted to demonstrate the effectiveness of the proposed MPC algorithm on the nonlinear dynamic model of the ship roll motion. The model of the wave-induced disturbance can be described by a stochastic process based on the PiersonMoskowitz spectrum. The simulation of the ship model is performed by setting the sea state to 5 which corresponds to rough sea conditions. The significant wave height is selected as $H_{s}=3.5 \mathrm{~m}$, damping constant is chosen as $\zeta_{w}=0.1$ and $w_{n}$ is taken as a zero-mean Gaussian white process noise with different standard deviations for different sea conditions. The simulation study was performed using the vessel model given in Li et al. (2016). The vessel has a design speed of 15 knots. The linearised nonlinear model (1) is sampled at a rate of $50 \mathrm{~ms}$ to obtain:

$$
x_{k+1}=\left[\begin{array}{cc}
0.9999 & 0.0492 \\
-0.0053 & 0.9673
\end{array}\right] x_{k}+\left[\begin{array}{l}
0.0001 \\
0.0022
\end{array}\right] u_{k}+\left[\begin{array}{l}
0 \\
1
\end{array}\right] d_{k}^{w}
$$

To implement the observer, the tuning parameters are selected thus: $W=1, A_{i}, B_{i}$ and $C_{i}$ are all identity matrices and $D_{i}=0$. To act as a benchmark for the proposed predictive 


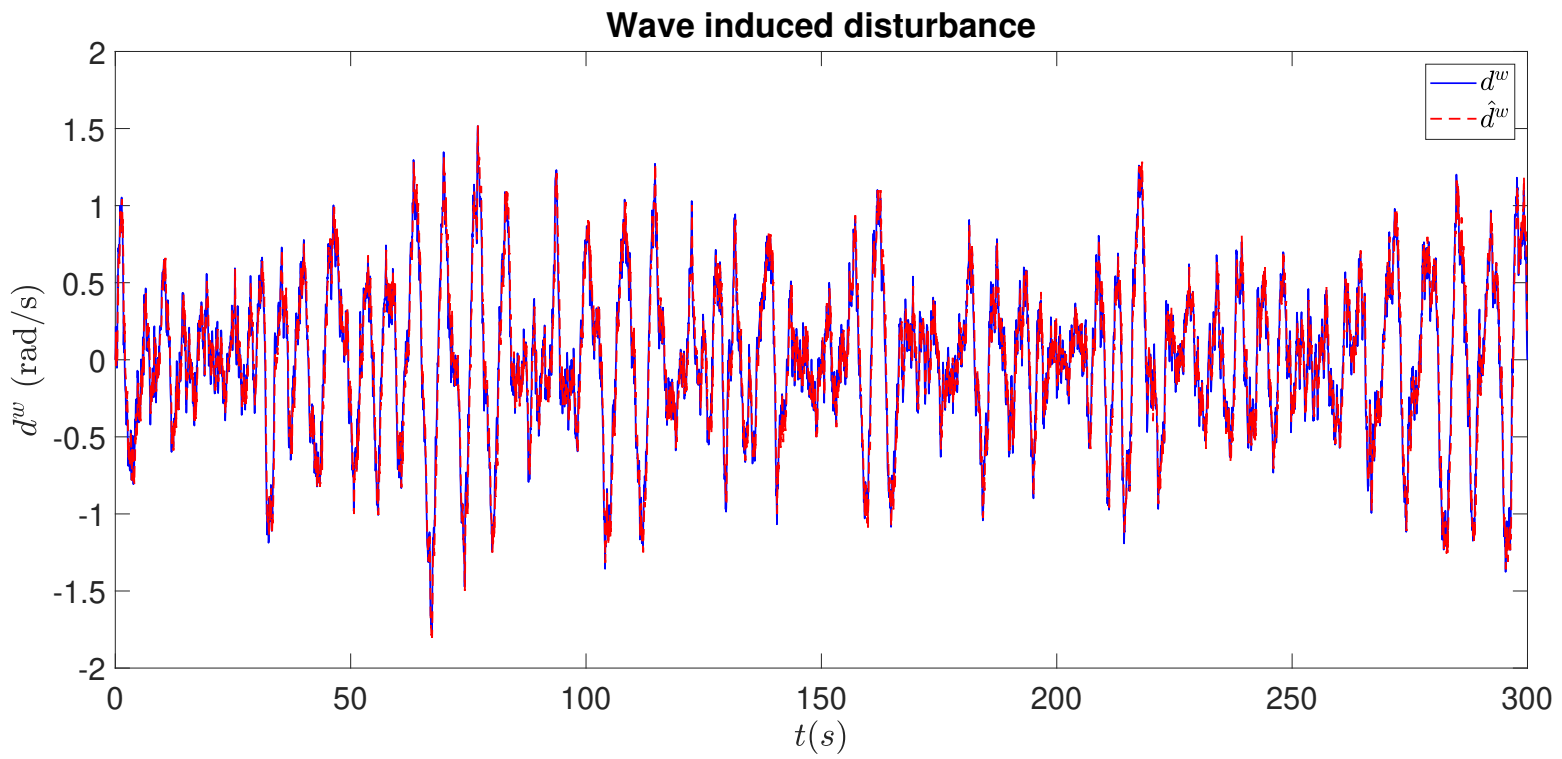

Figure 2: Time evolution of the wave-induced disturbance corresponding to beam sea conditions with significant sea height $H_{s}=3.5 \mathrm{~m}$ and its estimate are shown.

control algorithm, we designed an LQR controller by using the velocity model (10) to enable disturbance rejection. It is important to highlight that in LQR, the receding horizon control (RHC) problem is solved for $N=\infty$ while MPC is formulated to solve for finite $N$ to approximate infinite horizon controller. The open-loop performance is the response of the ship without active control. The tuning parameters used for the disturbance observer-based MPC (DOB-MPC) and LQR were selected as $Q=\operatorname{blkdiag}(5,0.01)$ and $R=0.02$. The terminal error weight $S$ will then be obtained from the solution of discrete-time Riccati equation. The parameters specific to the DOB-MPC were $P=10^{10}, N=100$. Due to the long prediction horizon $N$, we implemented a control horizon $N_{u}=2$ to speed up the solution to the optimisation problem and for improved performance. The simulation study was carried out in MATLAB environment. We present the simulation of the vessel in three different sea conditions.

\subsection{Beam Seas at Sea State 5}

As an illustration to show the nature of the disturbance, Figure 2 shows the wave-induced disturbance signal $d^{w}$ corresponding to rough sea state $5\left(H_{s}=3.5 \mathrm{~m}\right)$ with encounter angle, $\beta=90 \mathrm{deg}$ and its estimate $\hat{d}^{w}$ obtained by using the observer designed in Section 4 . The 
noise signal $w_{n}$ is taken as a Gaussian white noise with zero mean and standard deviation of 0.15 . It can be seen that the estimate closely follows the actual wave disturbance. In the following we would consider the controllers performance under two different forward ship speed.

\section{Case Study 1: Sea state 5 in beam seas with ship forward speed of 15 knots}

In this case study, we shall consider the simulation of the vessel when it sails at the design speed of 15 knots under the rough sea conditions. Figure 3 shows the plot of the nonlinear vessel model response without any constraint implementation in the system. In the plot, the dynamic states and control input amplitude and rate evolution with time for the open-loop, LQR and DOB-MPC simulation results are shown. The result shows that both LQR and DOB-MPC provide a significant roll motion damping when compared with the uncontrolled plant. The uncontrolled plant gives roll angle with a root mean square (RMS) value of $1.92 \mathrm{deg}$ and roll rate RMS value of $1.50 \mathrm{deg} / \mathrm{s}$. Whereas LQR reduced these values to $0.15 \mathrm{deg}$ and $0.36 \mathrm{deg} / \mathrm{s}$, DOB-MPC reduced the roll angle to $0.15 \mathrm{deg}$ and roll rate to $0.12 \mathrm{deg} / \mathrm{s}$, respectively. Therefore, the proposed controller achieved better roll angle and roll rate reduction which implies that the crew will enjoy a smoother sail under the DOB-MPC. The DOB-MPC provided a better performance because, in addition to compensating for disturbance amplitude via the velocity model, it compensates for the waveinduced disturbance rate.

Table I: Case Study 1 (Fin stabilised ship at 15 knots in beam seas): Performance comparison for the open loop, LQR and DOB-MPC assuming no physical limits on the input magnitude and rate constraints.

\begin{tabular}{llll}
\hline Parameter & Value $(\mathrm{RMS})$ & $\%$ Red & $\%$ Amp \\
\hline Roll angle open loop & 1.9214 & - & - \\
\hline Roll angle LQR & 0.1535 & $92.01 \%$ & - \\
\hline Roll angle DOB-MPC & 0.0911 & $95.26 \%$ & - \\
\hline Roll rate open loop & 1.4991 & - & - \\
\hline Roll rate LQR & 0.3632 & $75.77 \%$ & - \\
\hline Roll rate DOB-MPC & 0.1242 & $91.72 \%$ & \\
\hline
\end{tabular}



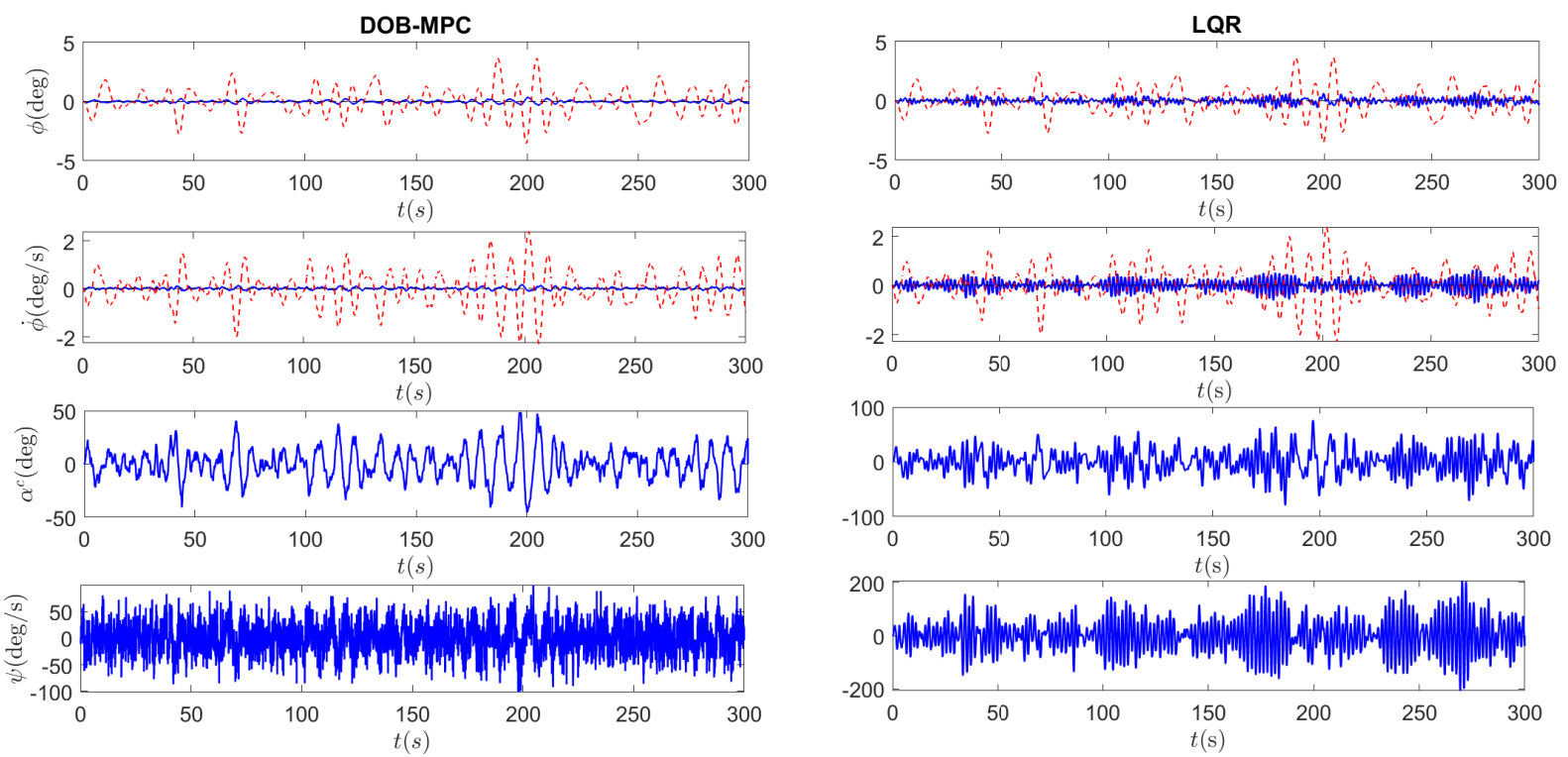

Figure 3: Case study 1 (Fin stabilised ship at 15 knots in beam seas): Time evolution of the vessel model states, input and input rate in the presence of wave-induced disturbance $d^{w}$ without input and input rate constraints. Open loop response (red) and the controlled plant response (blue) are shown.
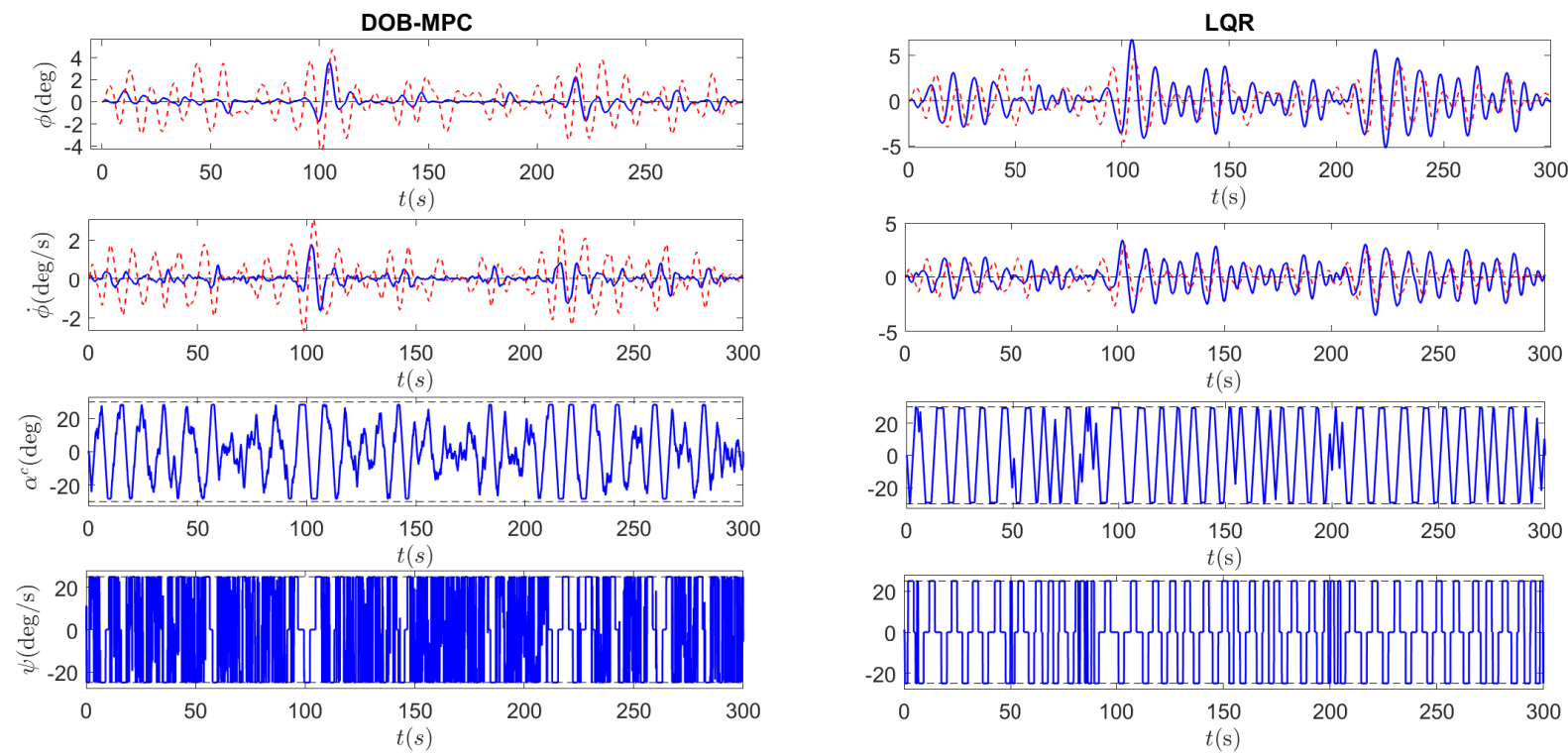

Figure 4: Case study 1 (Fin stabilised ship at 15 knots in beam seas): Time evolution of the vessel model states, input and input rate in the presence of wave-induced disturbance $d^{w}$ with input and input rate constraints. Open loop response (red) and the controlled plant response (blue) are shown. 
Table II: Case Study 1 (Fin stabilised ship at 15 knots in beam seas): Performance comparison for the open loop, LQR and DOB-MPC with input magnitude and rate constraints implemented.

\begin{tabular}{llll}
\hline Parameter & Value (RMS) & \% Red & \% Amp \\
\hline Roll angle open loop & 2.0847 & - & - \\
\hline Roll angle LQR & 2.8821 & - & $38.25 \%$ \\
\hline Roll angle DOB-MPC & 0.7565 & $63.71 \%$ & \\
\hline Roll rate open loop & 1.2623 & - & - \\
\hline Roll rate LQR & 2.6471 & - & $109.70 \%$ \\
\hline Roll rate DOB-MPC & 0.5039 & $60.08 \%$ & \\
\hline
\end{tabular}

However, the control input angles shown in Figure 3 are quite large and in practice, the input amplitude and rate are restricted and a typical range is $\pm 30 \mathrm{deg}$ (and $30 \mathrm{deg} / \mathrm{s}$ ). Hence, we considered a magnitude constraint of $\pm 30 \mathrm{deg}$ and maximum rate of $25 \mathrm{deg} / \mathrm{s}$. Figure 4 shows the simulation results for the constrained scenario. Based on the results, we found that the constraints caused LQR inputs and its rate to saturate. This caused roll angle and roll rate amplification with RMS values of $2.88 \mathrm{deg}$ and $2.65 \mathrm{deg} / \mathrm{s}$, respectively; which are higher than $2.08 \mathrm{deg}$ and $1.26 \mathrm{deg} / \mathrm{s}$ RMS values given by the open-loop plant. Under DOB-MPC control, the RMS values of the roll angle and roll rate are $0.76 \mathrm{deg}$ and $0.50 \mathrm{deg} / \mathrm{s}$ respectively. The results imply that DOB-MPC provides $63.71 \%$ reduction in roll and $60.08 \%$ decrease in the vessel roll rate while LQR gave $38.25 \%$ and $109.7 \%$ amplifications in roll angle and roll rate, respectively. In Tables I and II, the overall performances of the controllers in beam seas with ship's forward speed of 15 knots are summarised in terms of RMS values of the roll angle and roll rate errors.

\section{Case Study 2: Sea state 5 in beam seas with 10 knots ship Forward speed}

The use of fin stabiliser for roll motion stabilisation is well known to be especially effective at high speed (Hinostroza et al., 2015; Perez, 2006). In this case study, we shall examine the performance of the fin stabiliser when controlled by LQR and the proposed DOB-MPC at ship's speed that is two-third of design speed. The result of the simulation will give an idea of how effective the ship can be stabilised if it enters rough seas at a speed well below its rated forward speed. In Figure 5, the response obtained for the vessel at relatively low speed is 

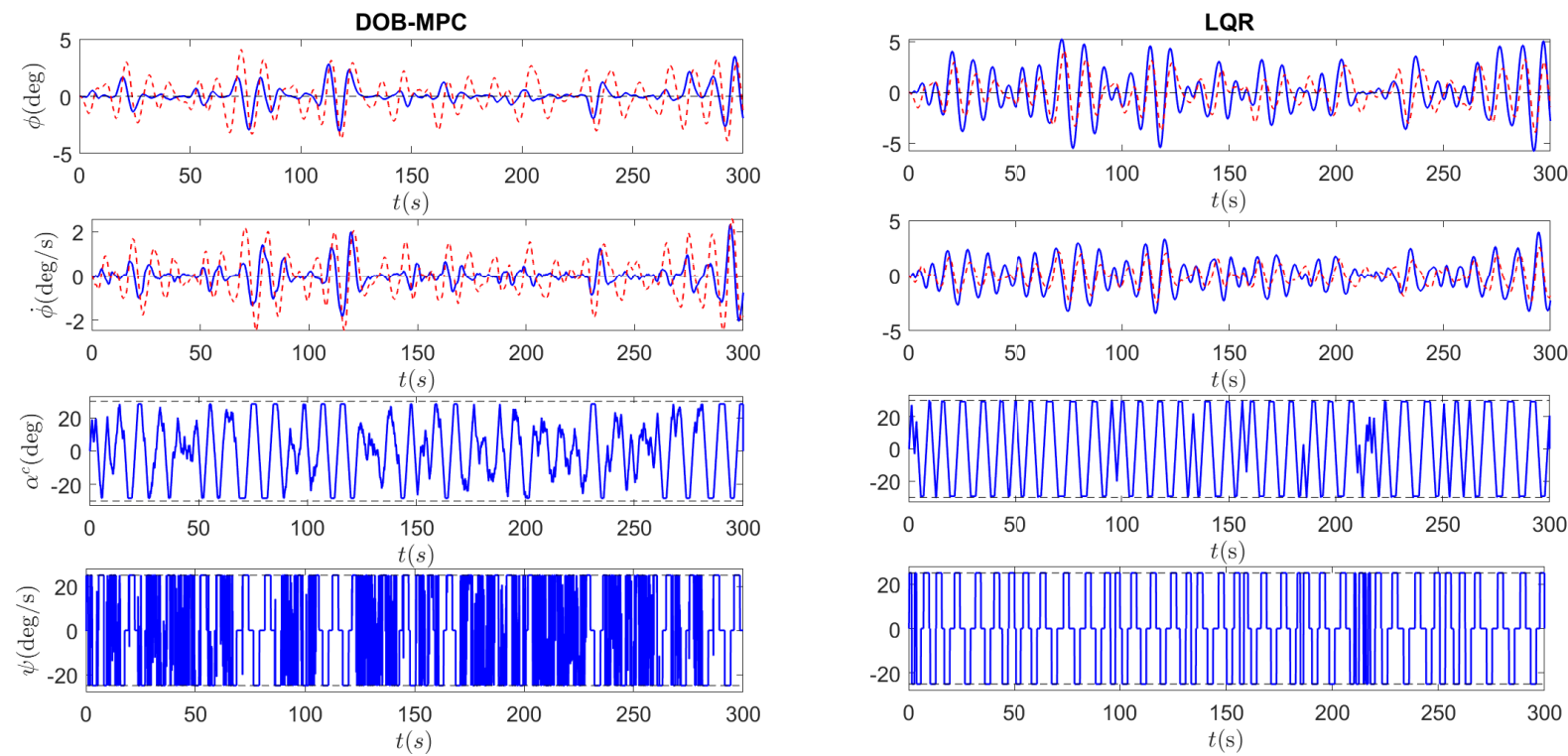

Figure 5: Case Study 2 (Fin stabilised ship at 10 knots in beam seas): Time evolution of the vessel model states, input and input rate in the presence of wave-induced disturbance $d^{w}$ with input and input rate constraints. Open loop response (red) and the controlled plant response (blue) are shown.

shown. In general, the results show that the proposed scheme is still able to lead to roll and roll rate reduction while LQR gave increased roll and its rate amplification. The result of the comparison of the performance of both control schemes is presented in Table III by computing the root mean square (RMS) values of the roll angle and roll rate errors. In comparison to the uncontrolled plant, the proposed DOB-MPC provided about $46 \%$ roll angle tracking error reduction while LQR gave 105\% roll tracking error amplification. Furthermore, LQR resulted in $122 \%$ roll rate error amplification while the proposed predictive controller provided $44 \%$ reduction of the tracking error in the roll rate. Therefore, for a reduced forward speed of the vessel, the proposed control scheme can provide a more comfortable sailing conditions in rough seas for the passengers on the ship when compared to the uncontrolled plant and LQR controlled plant.

\subsection{Quartering Seas at Sea State 5}

In this subsection, we consider rough sea conditions when the ship's encounter angle, $\beta=45 \mathrm{deg}$ (quartering seas). Here, the noise signal $w_{n}$ is assumed to have a standard deviation of $15 \%$. We consider different forward speed of the ship in the following two cases. 
Table III: Case Study 2 (Fin stabilised ship at 10 knots in beam seas): Performance comparison for the open loop, LQR and DOB-MPC responses with the input magnitude and rate constraints implemented.

\begin{tabular}{llll}
\hline Parameter & RMS & \% Red & \% Amp \\
\hline Roll angle open loop & 2.1248 & - & - \\
\hline Roll angle LQR & 3.7496 & - & $76.47 \%$ \\
\hline Roll angle DOB-MPC & 1.015 & $52.23 \%$ & - \\
\hline Roll rate open loop & 1.2153 & - & - \\
\hline Roll rate LQR & 2.7985 & - & $130.27 \%$ \\
\hline Roll rate DOB-MPC & 0.6422 & $47.16 \%$ & - \\
\hline
\end{tabular}

Table IV: Case Study 1 (Fin stabilised ship at 15 knots in quartering seas): Performance comparison for the open loop, LQR and DOB-MPC responses with the input magnitude and rate constraints implemented.

\begin{tabular}{llll}
\hline Parameter & Value $(\mathrm{RMS})$ & Reduction & Amplification \\
\hline Roll angle open loop & 3.3864 & - & - \\
\hline Roll angle LQR & 3.6935 & - & $9.07 \%$ \\
\hline Roll angle DOB-MPC & 0.8669 & $74.40 \%$ & - \\
\hline Roll rate open loop & 1.2473 & - & - \\
\hline Roll rate LQR & 1.6350 & - & $31.08 \%$ \\
\hline Roll rate DOB-MPC & 0.5333 & $57.24 \%$ & - \\
\hline
\end{tabular}

Case Study 1: Quartering seas at sea state 5 with 15 knots ship forward speed

The obtained simulation results are shown in Figure 6 where DOB-MPC is shown to give a significant reduction of roll and roll rate. As shown in Table IV, the proposed predictive control reduced the RMS error values of the roll rate and roll angle when compared to the open-loop plant by $74.4 \%$ and $57.24 \%$, respectively. LQR, on the other hand, gave $9.07 \%$ amplification in the vessel's roll and $31.08 \%$ roll rate amplification. Based on the tabulated results, it can be concluded that LQR generally led to roll motion amplification while the proposed control scheme resulted in a significant reduction of the ship's roll motion. 

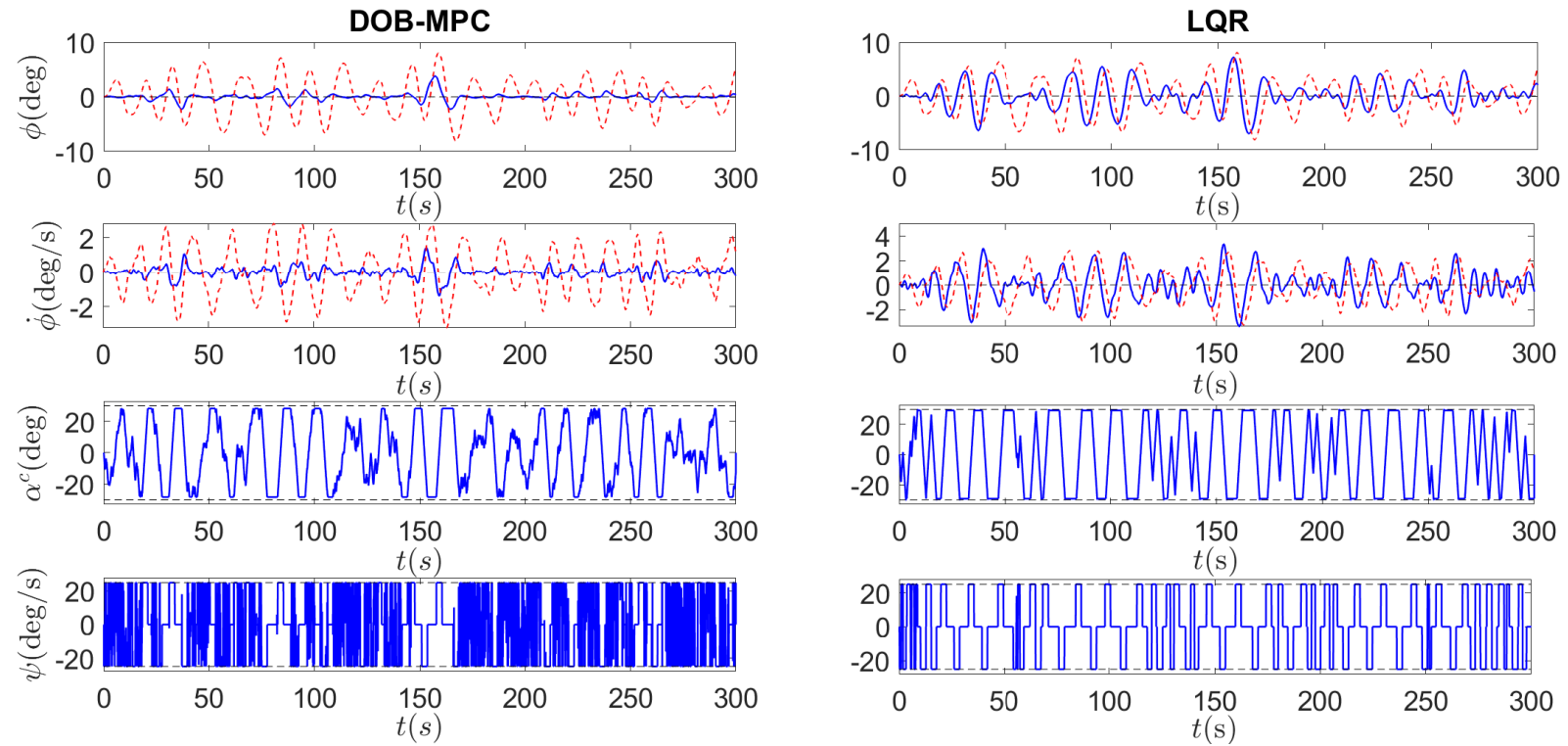

Figure 6: Case Study 1 (Fin stabilised ship at 15 knots in quartering seas): Time evolution of the vessel model states, input and input rate in the presence of wave-induced disturbance $d^{w}$ with input and input rate constraints. Open loop response (red) and the controlled plant response (blue) are shown.

\section{Case Study 2: Quartering seas at sea state 5 with ship forward speed of 10 knots}

The simulation results for the vessel at a reduced speed for sea state 5 in quartering seas is shown in Figure 7. Again, DOB-MPC is able to provide a significant performance improvement both in terms of roll angle and roll rate reduction. Whereas DOB-MPC reduced the RMS value of roll angle of the vessel from $3.22 \mathrm{deg}$ (uncontrolled) to $1.07 \mathrm{deg}$, LQR amplified the angle to achieve an RMS value of $4.45 \mathrm{deg}$. Furthermore, the overall reduction in roll and roll rate provided by DOB-MPC is demonstrated in Table V. LQR, on the other hand, generally resulted in amplification of roll angle and roll rate as shown in the table.

\subsection{Bow Seas at Sea State 5}

In this scenario, the wave-induced disturbance signal $d^{w}$ corresponds to rough sea state $5\left(H_{s}=3.5 \mathrm{~m}\right)$ with encounter angle, $\beta=135 \mathrm{deg}$ and its estimate $\hat{d}^{w}$. The noise signal $w_{n}$ is taken as a Gaussian white noise with zero mean and standard deviation of 0.4. Again, we shall consider the controllers performance under two different forward ship speed conditions. 
Table V: Case Study 2 (Fin stabilised ship at 10 knots in quartering Seas) : Performance comparison for the open loop, LQR and DOB-MPC responses with the input magnitude and rate constraints implemented.

\begin{tabular}{llll}
\hline Parameter & Value $(\mathrm{RMS})$ & Reduction & Amplification \\
\hline Roll angle open loop & 3.2196 & - & - \\
\hline Roll angle LQR & 4.4485 & - & $38.17 \%$ \\
\hline Roll angle DOB-MPC & 1.0691 & $66.79 \%$ & - \\
\hline Roll rate open loop & 1.4724 & - & - \\
\hline Roll rate LQR & 2.1790 & - & $47.99 \%$ \\
\hline Roll rate DOB-MPC & 0.5310 & $63.94 \%$ & - \\
\hline
\end{tabular}
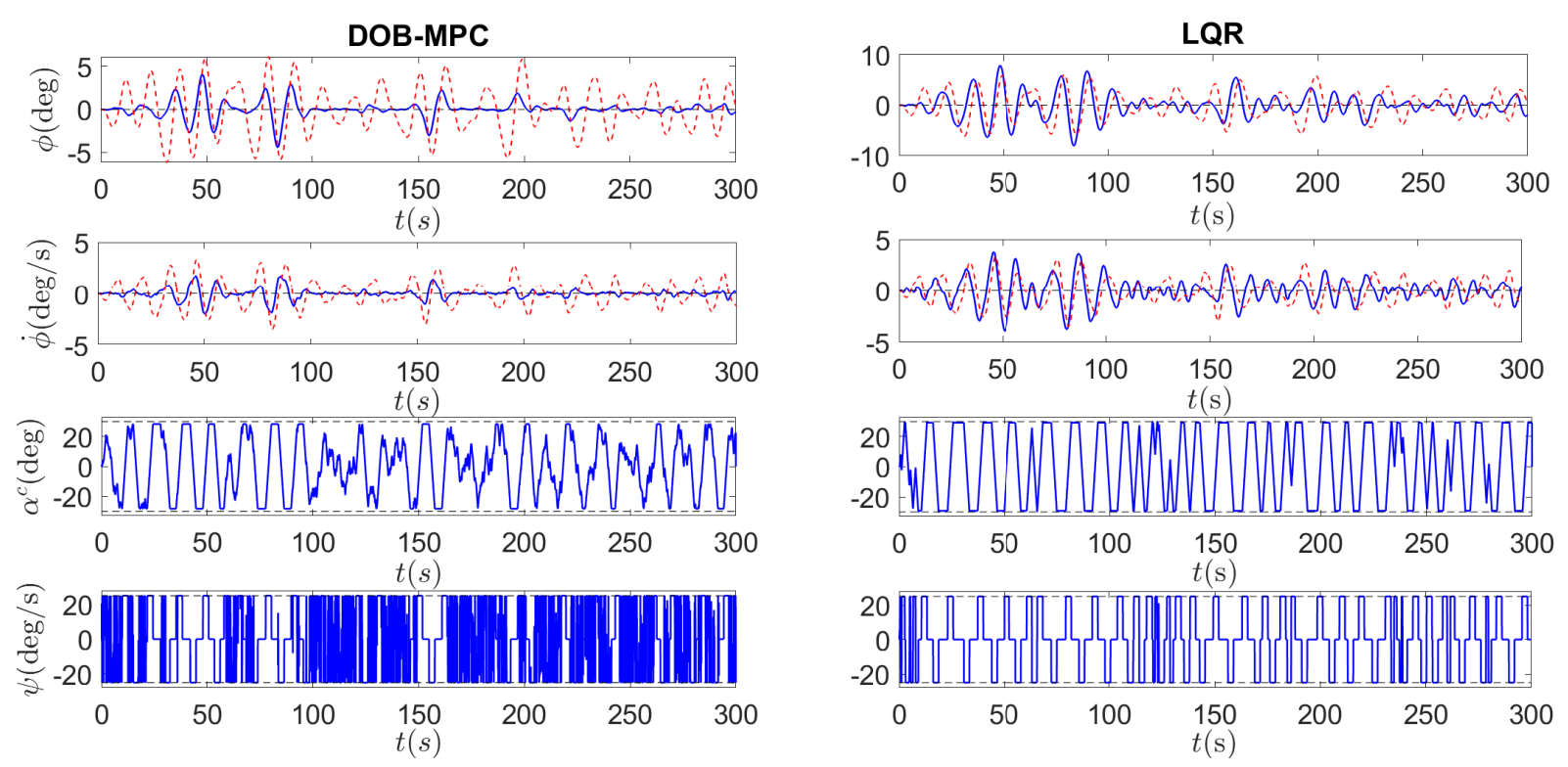
Table VI: Case Study 1 (Fin stabilised ship at 15 knots in bow seas): Performance comparison for the open loop, LQR and DOB-MPC responses with the input magnitude and rate constraints implemented.

\begin{tabular}{llll}
\hline Parameter & Value (RMS) & Reduction & Amplification \\
\hline Roll angle open loop & 0.9951 & - & - \\
\hline Roll angle LQR & 2.0649 & - & $107.51 \%$ \\
\hline Roll angle DOB-MPC & 0.4679 & $52.98 \%$ & - \\
\hline Roll rate open loop & 0.9472 & - & - \\
\hline Roll rate LQR & 2.5118 & - & $165.18 \%$ \\
\hline Roll rate DOB-MPC & 0.3832 & $59.54 \%$ & - \\
\hline
\end{tabular}

Case Study 1: Sea state 5 in bow seas with ship forward speed of 15 knots

The simulation results for the vessel with forward speed of 15 knots for sea state 5 in bow seas is shown in Figure 8. In the figure, minimal saturation occurred in the input signal of DOB-MPC compared to the control signal of LQR. However, the input rate saturation in the DOB-MPC is significantly greater than it is for LQR. Nonetheless, DOB-MPC still provide a significant performance improvement both in terms of roll angle and roll rate reduction. Whereas DOB-MPC reduced the output regulation RMS error of the vessel's roll angle from $1.00 \mathrm{deg}$ (uncontrolled) to $0.47 \mathrm{deg}$, LQR amplified this value to $2.06 \mathrm{deg}$. The improvement provided by DOB-MPC was even greater for roll rate while LQR provides greater amplification of the roll rate. The overall performances of LQR and DOB-MPC are presented in Table VI and LQR provided amplification of roll angle and roll rate.

Case Study 2: Sea state 5 in bow seas with 10 knots ship forward speed

The simulation results for the vessel's forward speed of 10 knots for sea state 5 in bow seas is shown in Figure 9. In the figure, DOB-MPC generally reduced the peak value of roll angle and its rate. In addition, DOB-MPC provides an overall significant performance improvement in the outputs regulation. Whereas DOB-MPC reduced the output regulation RMS error of the roll angle of the vessel from $1.45 \mathrm{deg}$ (uncontrolled) to $0.89 \mathrm{deg}$, LQR amplified this value to $3.66 \mathrm{deg}$. The improvement provided by DOB-MPC was even greater for roll rate while LQR provides greater amplification of the roll rate when compared to 
DOB-MPC
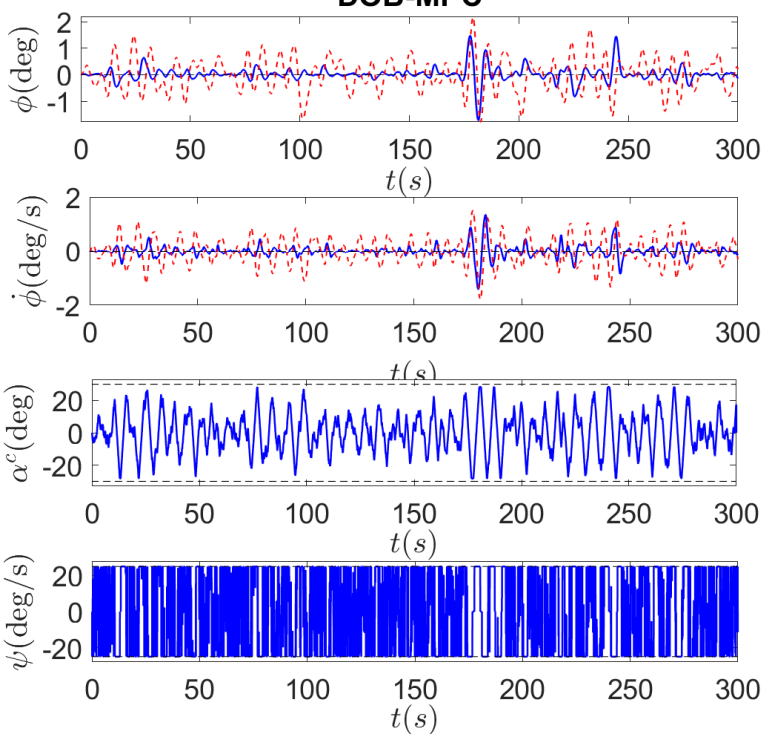

LQR
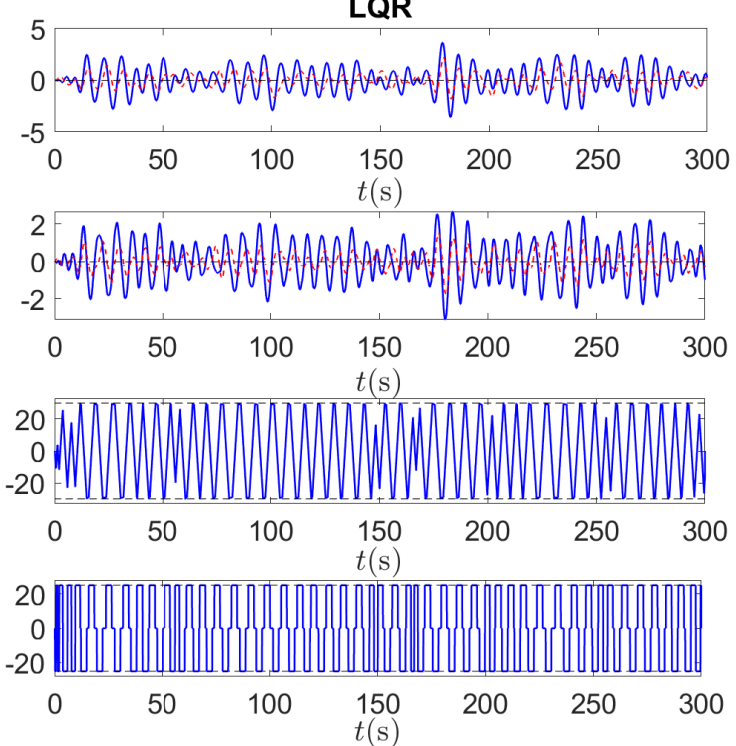

Figure 8: Case Study 1 (Fin stabilised ship at 15 knots in bow seas): Time evolution of the vessel model states, input and input rate in the presence of wave-induced disturbance $d^{w}$ with input and input rate constraints. Open loop response (red) and the controlled plant response (blue) are shown.

roll rate of the uncontrolled vessel. The overall reduction in roll and roll rate provided by DOB-MPC is demonstrated in Table VII; where LQR, on the other hand, generally resulted in amplification of roll motion.

\section{Discussions}

In this section, we shall discuss the results obtained from the numerical simulation study carried out in the previous section under various sea conditions and forward speed of the vessel. The study showed that, for the open loop response of the vessel, the maximum RMS value for roll was obtained in quartering seas when the forward speed of the vessel is 10 knots. The minimum roll angle, on the other hand, occurred in bow seas when the vessel forward speed is 15 knots. LQR and DOB-MPC were able to provide significant improvement in roll and roll rate reduction for an unconstrained fin actuator. It is pertinent to point out that, even in this case, DOB-MPC provided greater roll motion reduction and lesser sensitivity to the randomness to the sea waves. However, the unconstrained scenario is not practical; hence, we mainly considered constrained fin actuator is the simulation study. The results, obtained from the constrained input and input rate, showed that LQR mostly 
resulted in roll motion amplification while the proposed controller provided enormous roll motion reduction. In terms of vessel speed, roll motion reduction for the ship based on the control of the fin stabiliser was poorer at lower speed of the vessel; this is consistent with previous findings (Fossen, 2011; Perez, 2006). The proposed controller gave the best roll reduction in quartering seas (at high vessel speed) and the least in bow seas (at low vessel speed). In bow seas, where the encounter frequency is highest due to the angle of encounter, it is expected that the disturbances would have lesser impact on the roll motion of the vessel (Perez, 2006). This point is evident from the fact that the minimum peak values in roll motion was achieved under bow seas. However, the performances of both controllers were poorest in these sea conditions and this is because the controllers' performances are affected by other factors such as the speed of the vessel and the nature of $w_{n}$, the input white noise signal.

In general, the impressive performance of DOB-MPC is because the proposed control algorithm considers the input and rate constraint requirements in determining the 'best' control move that optimises the ship's response while LQR does not incorporate the constraints in the RHC problem. Also, the proposed scheme attenuates the wave-induced disturbance rate in addition to eliminating the disturbance magnitude. Moreover, the saturation of an LQR controller can generally lead to instability while MPC may not converge but would be restricted to oscillate in a limit circle. It is important to point out that increasing the gain $R$ in order to avoid saturation of the LQR control input leads to increased roll amplification. For instance, keeping $Q$ weight unchanged and choosing $R=500$ can help in the elimination of the saturation of the LQR controller. However, in beam seas at sea state 5 with vessel moving at rated forward speed, this settings can give roll angle amplification of up to $126 \%$ compared to the $109.7 \%$ shown in Table II. Hence, choosing the LQR parameters to avoid saturation of the control signal may worsen the performance of the control scheme in these challenging settings.

\section{Conclusions}

The ship roll motion has a nonlinear dynamics and is subject to sea wave-induced disturbances that threaten its stability. In this paper, an observer enhanced model predictive 
Table VII: Case Study 2 (Fin stabilised ship at 10 knots in bow seas): Performance comparison for the open loop, LQR and DOB-MPC responses with the input magnitude and rate constraints implemented.

\begin{tabular}{llll}
\hline Parameter & Value (RMS) & Reduction & Amplification \\
\hline Roll angle open loop & 1.4551 & - & - \\
\hline Roll angle LQR & 3.6561 & - & $151.26 \%$ \\
\hline Roll angle DOB-MPC & 0.8878 & $38.99 \%$ & - \\
\hline Roll rate open loop & 0.6294 & - & - \\
\hline Roll rate LQR & 1.6693 & - & $165.22 \%$ \\
\hline Roll rate DOB-MPC & 0.3928 & $37.59 \%$ & - \\
\hline
\end{tabular}
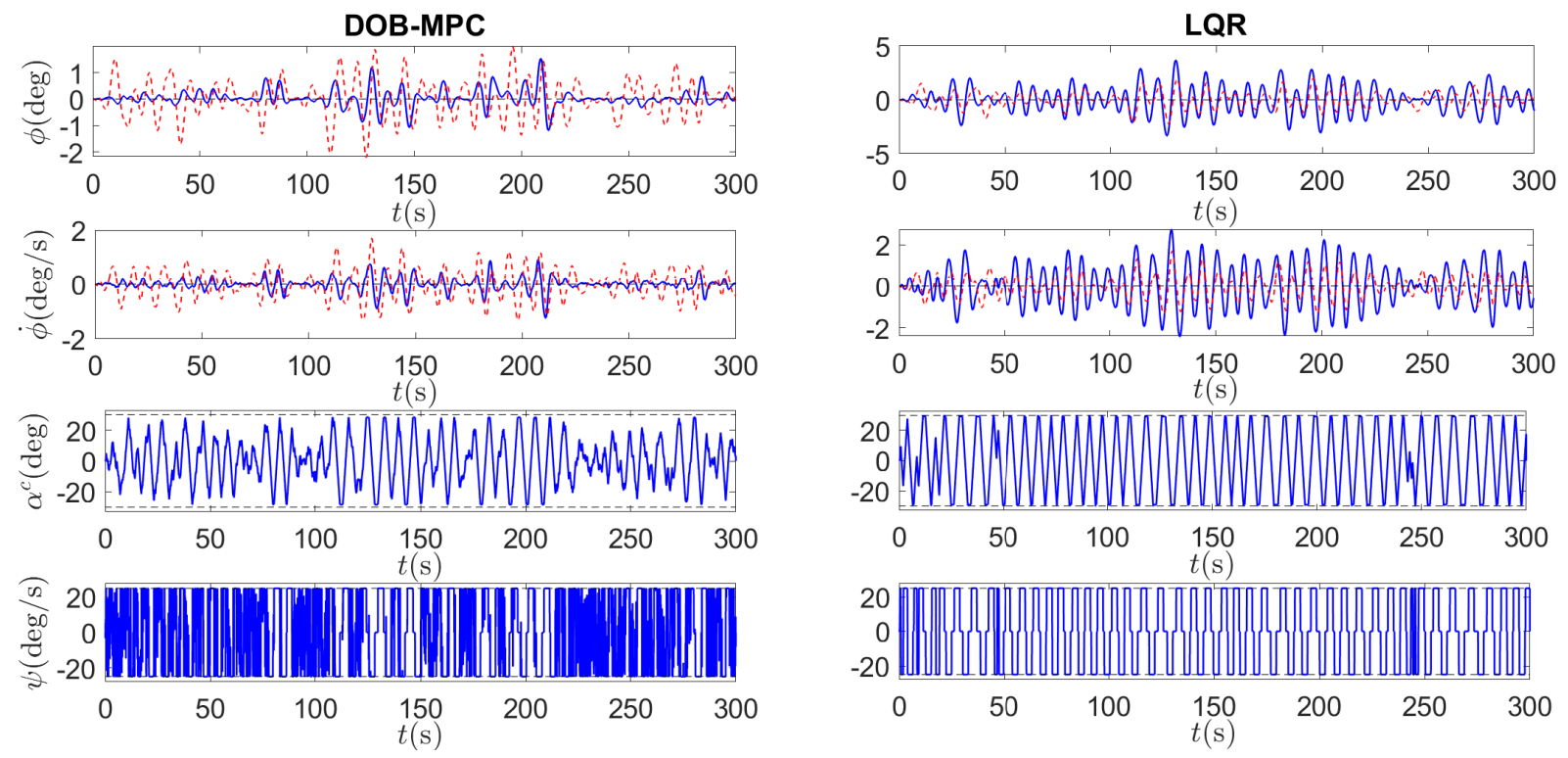
control algorithm based on velocity linear models with disturbance increment compensation has been proposed for the stabilisation of ship roll motion. Since the vessel model considered is nonlinear, we linearised the model around its operating point to obtain the proposed linear predictive controller. The study considers the environmental disturbances induced by waves and constraints on the fin actuator magnitude and rate. The proposed controller was designed by proposing a quadratic problem that computes the optimal input and disturbance increments. To ensure that the system was driven according to the minimisation of the cost function, the computed optimal control and disturbance deviations are used in every time step. This approach provides an additional degree of freedom for the control signal, which was exploited to formulate a control signal that is a function of the estimated environmental disturbances. By ensuring that the estimated wave-induced disturbance increment is always in opposition to the actual disturbance increment, the effects of the wave-induced disturbances were further mitigated. To estimate the environmental disturbances induced by waves, we designed an observer whose gain was obtained by solving an $\mathcal{H}_{2}$ minimisation problem. Furthermore, the stability analysis of the control system was provided. Numerical simulation of the vessel in various rough sea conditions showed that the proposed algorithm can give significant performance improvement in ship roll motion stabilisation even when the ship's forward speed is lower than its rated speed.

In this study, we considered the constraints in the input and its rate without considering the effective angle of attack which is usually important in a design situation. In future research, the effective angle of attack may be considered to prevent a dynamic stall in the fins. Also, it would be interesting to explore the performance of the control method when there is a change in sailing conditions such as a sudden change in sea state or direction of sail while the ship is in motion. Furthermore, whereas our control method relied on a single step estimation and direct attenuation of the wave-induced disturbance rate, future research may investigate an alternative means that rely on predicted disturbance rate over a defined horizon based on its current estimate to compensate for the rate of change of the environmental disturbances. 


\section{Acknowledgment}

The first author is thankful to the Commonwealth Scholarship Commission (CSC) in the UK for sponsoring, under Award Number NGSS-2018-73, his MSc degree at Glasgow Caledonian University (GCU) and providing him with a thesis research grant.

\section{References}

Bai, R., 2014. Adaptive fuzzy output-feedback method applied to fin control for time-delay ship roll stabilization. Mathematical Problems in Engineering 2014.

Bai, W., Li, T., Gao, X., Myint, K.T., 2013. Neural network based direct adaptive backstepping method for fin stabilizer system, in: International Symposium on Neural Networks, Springer. pp. 212-219.

Bassler, C.C., Reed, A.M., 2009. An analysis of the bilge keel roll damping component model, in: Proc. 10th Intl. Conf. Stability of Ships and Ocean Vehicles.

Crossland, P., 2003. The effect of roll-stabilisation controllers on warship operational performance. Control Engineering Practice 11, 423-431.

Fortuna, L., Muscato, G., 1996. A roll stabilization system for a monohull ship: modeling, identification, and adaptive control. IEEE Transactions on control systems technology 4 , $18-28$.

Fossen, T.I., 2011. Handbook of marine craft hydrodynamics and motion control. John Wiley \& Sons.

Grimm, G., Messina, M.J., Tuna, S.E., Teel, A.R., 2004. Examples when nonlinear model predictive control is nonrobust. Automatica 40, 1729-1738.

Hickey, N., Grimble, M., Johnson, M., Katebi, M., Melville, R., 1997. Robust fin roll stabilisation of surface ships, in: Proceedings of the 36th IEEE Conference on Decision and Control, IEEE. pp. 4225-4230. 
Hickey, N., Grimble, M., Johnson, M., Katebi, R., Wood, D., 1995. H-infinity fin roll stabilisation control system design. IFAC Proceedings Volumes 28, 304-311.

Hinostroza, M., Luo, W., Soares, C.G., 2015. Robust fin control for ship roll stabilization based on 12-gain design. Ocean Engineering 94, 126-131.

Irkal, M.A., Nallayarasu, S., Bhattacharyya, S., 2019. Numerical prediction of roll damping of ships with and without bilge keel. Ocean Engineering 179, 226-245.

Jimoh, I.A., Küçükdemiral, I.B., Bevan, G., Orukpe, P.E., 2020. Offset-free model predictive control: A study of different formulations with further results, in: 2020 28th Mediterranean Conference on Control and Automation (MED), IEEE. pp. 671-676.

Kucukdemiral, I.B., Cakici, F., Yazici, H., 2019. A model predictive vertical motion control of a passenger ship. Ocean Engineering 186, 106100.

Kula, K., 2015. An overview of roll stabilizers and systems for their control. TransNav: International Journal on Marine Navigation and Safety of Sea Transportation 9.

Kuseyri, S., 2020. Constrained h $\infty$ control of gyroscopic ship stabilization systems. Proceedings of the Institution of Mechanical Engineers, Part M: Journal of Engineering for the Maritime Environment, 1475090220903217.

Lee, S., Rhee, K.P., Choi, J.W., 2011. Design of the roll stabilization controller, using fin stabilizers and pod propellers. Applied Ocean Research 33, 229-239.

Li, R., Li, T., Bai, W., Du, X., 2016. An adaptive neural network approach for ship roll stabilization via fin control. Neurocomputing 173, 953-957.

Liu, C., Wang, D., Zhang, Y., Meng, X., 2020. Model predictive control for path following and roll stabilization of marine vessels based on neurodynamic optimization. Ocean Engineering 217, 107524.

Liu, J., Allen, R., Yi, H., 2011. Ship motion stabilizing control using a combination of model predictive control and an adaptive input disturbance predictor. Proceedings of the 
Institution of Mechanical Engineers, Part I: Journal of Systems and Control Engineering 225, 591-602.

Malekizade, H., Jahed-Motlagh, M.R., Moaveni, B., Moarefianpour, A., Ghassemi, H., 2016. Robust model predictive control employed to the container ship roll motion using finstabilizer. Cogent Engineering 3, 1235478.

Pannocchia, G., Gabiccini, M., Artoni, A., 2015. Offset-free mpc explained: novelties, subtleties, and applications. IFAC-PapersOnLine 48, 342-351.

Pannocchia, G., Rawlings, J.B., 2003. Disturbance models for offset-free model-predictive control. AIChE journal 49, 426-437.

Pannocchia, G., Rawlings, J.B., Wright, S.J., 2011a. Conditions under which suboptimal nonlinear mpc is inherently robust. Systems \& Control Letters 60, 747-755.

Pannocchia, G., Wright, S.J., Rawlings, J.B., 2011b. Partial enumeration mpc: Robust stability results and application to an unstable cstr. Journal of Process Control 21, 14591466.

Pascoal, R., Rodrigues, B., Soares, C.G., 2005. Roll-yaw regulation using stabilizing fins and rudder in a disturbance observer based compensator scheme, in: Maritime Transportation and Exploitation of Ocean and Coastal Resources, Two Volume Set. CRC Press, pp. 740747.

Perez, T., 2006. Ship motion control: course keeping and roll stabilisation using rudder and fins. Springer Science \& Business Media.

Perez, T., Blanke, M., 2012. Ship roll damping control. Annual Reviews in Control 36, $129-147$.

Perez, T., Goodwin, G.C., 2008. Constrained predictive control of ship fin stabilizers to prevent dynamic stall. Control Engineering Practice 16, 482-494. 
Pierson Jr, W.J., Moskowitz, L., 1964. A proposed spectral form for fully developed wind seas based on the similarity theory of sa kitaigorodskii. Journal of geophysical research $69,5181-5190$.

Scherer, C., Weiland, S., 2015. Linear matrix inequalities in control. Lecture Notes, Dutch Institute for Systems and Control, Delft, The Netherlands 3.

Sharif, M., Roberts, G., Sutton, R., 1995. Sea-trial experimental results of fin/rudder roll stabilisation. Control Engineering Practice 3, 703-708.

Sun, M., Luan, T., Liang, L., 2018. Ocean Engineering 163, 307-321.

Surendran, S., Kiran, V., 2007. Control of ship roll motion by active fins using fuzzy logic. Ships and Offshore Structures 2, 11-20.

Surendran, S., Lee, S., Kim, S., 2007. Studies on an algorithm to control the roll motion using active fins. Ocean Engineering 34, 542-551.

Sutton, R., Roberts, G., Dearden, S., 1990. Warship roll stabilisation using fuzzy control of the fin stabilisers, in: Advanced Information Processing in Automatic Control (AIPAC'89). Elsevier, pp. 171-175.

Wang, H., Chen, B., Lin, C., 2012. Direct adaptive neural control for strict-feedback stochastic nonlinear systems. Nonlinear Dynamics 67, 2703-2718.

Wang, L., Xiros, N.I., Loghis, E.K., 2019. Design and comparison of Ho/H2 controllers for frigate rudder roll stabilization. Journal of Marine Science and Application 18, 492-509. 